\title{
POD-Based Mixed-Integer Optimal Control of the Heat Equation
}

\author{
Bachmann, Freya · Beermann, Dennis · Lu, Jianjie · Volkwein, Stefan
}

\begin{abstract}
In the present paper an optimal control problem governed by the heat equation is considered, where continuous as well as discrete controls are involved. To obtain the discrete controls the branch-and-bound method is utilized, where in each node a relaxed control constrained optimal control problem has to be solved involving only continuous controls. However, the solutions to many relaxed optimal control problems have to be computed numerically. For that reason model-order reduction is applied to speed-up the branch-and-bound method. In this work the method of proper orthogonal decomposition (POD) is used. A posteriori error estimation in each node ensures that the calculated solutions are sufficiently accurate. Numerical experiments illustrate the efficiency of the proposed strategy.
\end{abstract}

\section{Introduction}

In the present article an optimal control problem is considered which is motivated by energy efficient building operation. Energy efficiency is nowadays one driving force for achieving innovations in improved system and process operation. Within the European Union, the building sector is the largest energy consumer accounting for $40 \%$ of the overall energy consumption with a fraction of $60 \%$ referring to residential buildings [7]. Similar numbers are available for the United States, where buildings contribute to about $40 \%$ of the total energy expenditure with a 55\% fraction accounting for residential buildings. According to the National Science and Technology Council [27], a 70\% reduction in building energy consumption is equivalent to the elimination of the

\section{S. Volkwein}

Department of Mathematics and Statistics, University of Konstanz, Universitätstraße 10, D-78457 Konstanz, Germany, E-mail: stefan.volkwein@uni-konstanz.de total energy expenditure of the US transport sector. Assuming persistent growth rates it can be expected that buildings will by 2025 be the largest world wide energy consumer and will by 2050 surpass the energy consumption of industry and transportation [27,32]. During the life cycle of a building, $84 \%$ of provided energy are consumed for the building operation ( $12 \%$ for construction, $4 \%$ for maintenance) [31]. As a result, the proper adjustment of the building operation enables a significant saving of primary and secondary energy. For this, automation, control and mathematical optimization are identified as key ingredients to achieve energy efficiency [9].

Motivated by the importance of energy efficient building operation, we are discussing a related, but simplified problem in the present paper. The goal is to reach a certain desired temperature distribution in a room while choosing proper insulation materials for the wall boundaries as well as an optimal underfloor heating strategy. The temperature is governed by the heat equation, where we do not include convection for the moment. Since the insulation material has to be chosen from a discrete set and the heating is described by a time-continuous control, the optimal control involves continuous and discrete controls. These kind of problems are considered in $[10,11,23]$, for instance, for ordinary differential equations. For partial differential equations (PDEs) we refer to the note [20]. In particular, mixed-integer problems for hyperbolic PDEs are considered, e.g., for problems for gas transportation systems [14] and traffic flow [15,16].

The new contribution of this work is the application of reduced-order modeling, which has turned out to be an efficient method for PDE-constrained optimization; cf. [4,22, 24]. We apply the method of proper orthogonal decomposition (POD) to derive reduced-order models for the PDEconstrained mixed-integer optimal control problem. To ensure the accuracy of the reduced-order approximation, a posteriori error estimation is utilized. Here, we can rely on pre- 
vious work; $\operatorname{cf}[12,13,26,29]$. In order to tackle numerically the mixed-integer, time-dependent constraints, a branch-andbound algorithm was implemented; see [19] and, e.g., [30]. The idea is based on solving a set of correlated relaxation problems by omitting the integrality conditions and computing numerically many optimal control problems involving only continuous free variables.

The paper is organized as follows: In Section 2 we introduce the mixed-integer optimal control problem and review some theoretical results. The optimal control problem can be formulated as a pure control constrained minimization problem. This is explained in Section 3. For the numerical solution a Galerkin discretization is introduced in Section 4. In Section 5 the reduced-order modeling based on POD is described, while the branch-and-bound method is discussed in Section 6. Section 7 is devoted to numerical experiments utilizing real weather data from Constance (Germany) and Lisbon (Portugal). Finally, we draw conclusions in the last section.

Notation: For two vectors $x^{1}, x^{2} \in \mathbb{R}^{n}$, we write $x^{1} \leq x^{2}$ if $x_{i}^{1} \leq x_{i}^{2}$ holds for $i=1, \ldots, n$. For two functions $f^{1}, f^{2} \in$ $L^{p}\left(0, T ; \mathbb{R}^{n}\right)$ with $p \in[1, \infty]$, we write $f^{1} \leq f^{2}$ if $f^{1}(t) \leq$ $f^{2}(t)$ holds in $\mathbb{R}^{n}$ for almost all $t \in(0, T)$.

\section{Problem formulation}

We consider a (horizontal cross-section of a) room represented by a domain $\Omega \subset \mathbb{R}^{d}(d \in\{2,3\})$ over a time period $(0, T)$ with final time $T>0$. Let $\Omega$ as well as its boundary $\Gamma=\partial \Omega$ be split into disjoint subdomains, i.e.,

$\Omega=\bigcup_{j=1}^{N^{\mathrm{c}}} \Omega_{j} \quad$ and $\quad \Gamma=\bigcup_{j=0}^{N^{\mathrm{i}}} \Gamma_{j}$.

In (1) each $\Omega_{j}\left(j=1, \ldots, N^{\mathrm{c}}\right)$ represents a portion of the building that can be heated individually over time, whereas each $\Gamma_{j}\left(j=1, \ldots, N^{\mathrm{i}}\right)$ is a portion of an exterior wall which has to be equipped with insulation material. The boundary set $\Gamma_{0}$ represents the interior walls of the buildings. Let $Q:=$ $(0, T) \times \Omega$ denote the space-time cuboid, $y=y(t, x)$ the temperature inside the building and $\Sigma:=(0, T) \times \Gamma$. For the definition of Lebesgue and Sobolev spaces we refer the reader to [8], for instance. We introduce the problem of maintaining a certain desired temperature $y_{d} \in L^{2}(Q)$ as closely as possible while at the same time minimizing the heating and insulation costs:

$$
\begin{aligned}
J\left(y, u^{\mathrm{c}}, u^{\mathrm{i}}\right) & =\frac{\alpha^{Q}}{2} \iint_{Q}\left|y(t, x)-y_{d}(t, x)\right|^{2} \mathrm{~d} x \mathrm{~d} t \\
& +\frac{1}{2} \sum_{j=1}^{N^{\mathrm{c}}} \alpha_{j}^{\mathrm{c}} \int_{0}^{T}\left|u_{j}^{\mathrm{c}}(t)\right|^{2} \mathrm{~d} t+\frac{1}{2} \sum_{j=1}^{N^{\mathrm{i}}} \alpha_{j}^{\mathrm{i}}\left|u_{j}^{\mathrm{i}}-\hat{u}_{j}^{\mathrm{i}}\right|^{2}
\end{aligned}
$$

Here,

$$
u^{\mathrm{c}}=\left(u_{1}^{\mathrm{c}}, \ldots, u_{N^{\mathrm{c}}}^{\mathrm{c}}\right) \in L^{2}\left(0, T ; \mathbb{R}^{N^{\mathrm{c}}}\right)
$$

and

$u^{\mathrm{i}}=\left(u_{1}^{\mathrm{i}}, \ldots, u_{N^{\mathrm{i}}}^{\mathrm{i}}\right)$

are the controls of the system and stand for the heating over time and certain choices of insulation material at the wall, respectively. The parameters $\alpha^{\mathrm{c}} \in \mathbb{R}_{+}^{N^{\mathrm{c}}}$ and $\alpha^{\mathrm{i}} \in \mathbb{R}_{+}^{N^{\mathrm{i}}}$ are the costs associated with these controls, where we set $\mathbb{R}_{+}:=$ $\{s \in \mathbb{R} \mid s>0\}$. The reference control $\hat{u}^{\mathrm{i}} \in \mathbb{R}^{N^{\mathrm{i}}}$ represents the cheapest insulation material we could possibly choose. It will become apparent after the model has been introduced that smaller values of $u^{\mathrm{i}}$ correspond to better insulation material, so we will only admit controls which are componentwise smaller than $\hat{u}^{i}=u_{\mathrm{b}}^{\mathrm{i}}$ and penalize the deviation from this reference control. The non-negative scalar $\alpha^{Q}$ represents the significance we attribute to the temperature with respect to the investment costs and can be chosen to weight these two incomparable factors against each other.

The room temperature is described in a simplified manner by a linear heat equation:

$$
\begin{aligned}
y_{t}(t, x)-\kappa \Delta y(t, x) & =\sum_{j=1}^{N^{\mathrm{c}}} u_{j}^{\mathrm{c}}(t) \chi_{j}^{\mathrm{c}}(x), & & (t, x) \in Q \\
\kappa \frac{\partial y}{\partial n}(t, s) & =\sum_{j=1}^{N^{\mathrm{i}}} u_{j}^{\mathrm{i}} \chi_{j}^{\mathrm{i}}(t, s), & & (t, s) \in \Sigma \\
y(0, x) & =y_{\circ}(x), & & x \in \Omega
\end{aligned}
$$

Here, $\kappa \in \mathbb{R}_{+}$denotes a diffusion coefficient. The shape functions $\chi_{j}^{\mathrm{c}} \in L^{\infty}(\Omega)\left(j=1, \ldots, N^{\mathrm{c}}\right)$ take non-zero values only on the subdomain $\Omega_{j}$. Similarly, the functions $\chi_{j}^{\mathrm{i}} \in$ $L^{\infty}(\Sigma)\left(j=1, \ldots, N^{\mathrm{i}}\right)$ take non-zero values on the boundary sets $\Sigma_{j}:=(0, T) \times \Gamma_{j}\left(j=1, \ldots, N^{\mathrm{i}}\right)$. The latter need to be time-dependent in order to model the transient behavior of the outside temperature. Note that we have imposed homogeneous Neumann boundary conditions on the interior walls $\Gamma_{0}$, which translates to ideal insulation. Finally, we assume that $y_{\circ} \in L^{\infty}(\Omega)$ is a given initial temperature.

In addition to (2a) and ( $2 \mathrm{~b})$, we introduce bilateral constraints for the controls. We choose the control space $\mathcal{U}:=$ $L^{2}\left(0, T ; \mathbb{R}^{N^{\mathrm{c}}}\right) \times \mathbb{R}^{N^{\mathrm{i}}}$ endowed by the usual Hilbert space product topology. Furthermore, we are given lower and upper bounds $u_{\mathrm{a}}^{\mathrm{c}}, u_{\mathrm{b}}^{\mathrm{c}} \in L^{2}\left(0, T ; \mathbb{R}^{N^{\mathrm{c}}}\right)$ and $u_{\mathrm{a}}^{\mathrm{i}}, u_{\mathrm{b}}^{\mathrm{i}} \in \mathbb{R}^{N^{\mathrm{i}}}$ with $u_{\mathrm{a}}^{\mathrm{c}} \leq u_{\mathrm{b}}^{\mathrm{c}}$ and $u_{\mathrm{a}}^{\mathrm{i}} \leq u_{\mathrm{b}}^{\mathrm{i}}$, respectively. Then, we demand that

$$
\left(u^{\mathrm{c}}, u^{\mathrm{i}}\right) \in \mathcal{U}_{\mathrm{ad}}\left(u_{\mathrm{a}}^{\mathrm{i}}, u_{\mathrm{b}}^{\mathrm{i}}\right)
$$

where the admissible set is defined as

$$
\mathcal{U}_{\mathrm{ad}}(\mathrm{a}, \mathrm{b})=\left\{\left(v^{\mathrm{c}}, v^{\mathrm{i}}\right) \in \mathcal{U} \mid u_{\mathrm{a}}^{\mathrm{c}} \leq v^{\mathrm{c}} \leq u_{\mathrm{b}}^{\mathrm{c}}, \mathrm{a} \leq v^{\mathrm{i}} \leq \mathrm{b}\right\}
$$


for vectors $\mathrm{a}, \mathrm{b} \in \mathbb{R}^{N^{\mathrm{i}}}$ satisfying $\mathrm{a} \leq \mathrm{b}$. We incorporate the bounds for the boundary controls $u^{i}$ into the notation of the admissible set because these will vary throughout the branchand-bound method. This is due to the fact that we consider the case, where there is only a discrete number of insulation materials to choose from for the controls $u^{\mathrm{i}}$. We represent this by the following integrality constraint for the boundary variable:

$\left(u^{\mathrm{c}}, u^{\mathrm{i}}\right) \in \mathcal{U}^{\mathrm{int}}:=\left\{\left(v^{\mathrm{c}}, v^{\mathrm{i}}\right) \in \mathcal{U} \mid v^{\mathrm{i}} \in \mathbb{Z}^{N^{\mathrm{i}}}\right\}$

We call (2a)-(2d) the Mixed-Integer Optimal Control Problem (MIOCP).

\section{The reduced optimal control problem}

We introduce the spaces $H:=L^{2}(\Omega)$ and $V:=H^{1}(\Omega)$ which form a Gelfand triple $V \hookrightarrow H=H^{\prime} \hookrightarrow V^{\prime}$, where each embedding is continuous and dense; cf. [8]. It is well-known [6] that the space

$W(0, T)=L^{2}(0, T ; V) \cap H^{1}\left(0, T ; V^{\prime}\right)$

is a Hilbert space endowed with the inner product

$\langle\varphi, \phi\rangle_{W(0, T)}=\int_{0}^{T}\left\langle\varphi_{t}(t), \phi_{t}(t)\right\rangle_{V^{\prime}}+\langle\varphi(t), \phi(t)\rangle_{V} \mathrm{~d} t$

for $\varphi, \phi \in V$. We call $y \in W(0, T)$ a weak solution to (2b) if it holds for all $\varphi \in V$ and almost all $t \in(0, T)$ :

$$
\begin{aligned}
& \left\langle y_{t}(t), \varphi\right\rangle_{V^{\prime} \times V}+\kappa \int_{\Omega} \nabla y(t, x) \cdot \nabla \varphi(x) \mathrm{d} x= \\
& \sum_{j=1}^{N^{\mathrm{c}}} u_{j}^{\mathrm{c}}(t) \int_{\Omega} \chi_{j}^{\mathrm{c}}(x) \varphi(x) \mathrm{d} x+\sum_{j=1}^{N^{\mathrm{i}}} u_{j}^{\mathrm{i}} \int_{\Gamma} \chi_{j}^{\mathrm{i}}(t, s) \varphi(s) \mathrm{d} s
\end{aligned}
$$

as well as

$y(0)=y_{\circ} \quad$ in $H$

In (3a) we denote by $\langle\cdot, \cdot\rangle_{V^{\prime} \times V}$ the dual pairing between $V$ and its dual space $V^{\prime}$. It is known $[6,28]$ that (3) admits a unique solution $y \in y:=W(0, T)$ for every control $u=\left(u^{\mathrm{c}}, u^{\mathrm{i}}\right) \in \mathcal{U}$. Moreover, there is an affine-linear, continuous control-to-state operator $\mathcal{S}: \mathcal{U} \rightarrow \mathcal{y}$, where $y=\mathcal{S} u$ solves (3) for $u \in \mathcal{U}$. The operator $\mathcal{S}$ has the structure $\mathcal{S} u=\mathcal{S}_{0} u+\hat{y}$, where $S_{0}: \mathcal{U} \rightarrow \mathcal{y}$ is a linear, bounded operator which maps a control $u \in \mathcal{U}$ to the solution of (3) with $y_{\circ}=0$. Further, $\hat{y} \in W(0, T)$ is the solution to (3) with $u=0$.

The introduction of the control-to-state operator $\mathcal{S}$ and the admissible set $\mathcal{U}_{\mathrm{ad}}$ allows us to rewrite (MIOCP) as a minimization problem in the control variable only:

$\min \hat{J}(u):=J(\mathcal{S} u, u)$ subject to (s.t.) $u \in \mathcal{U}_{\mathrm{ad}}^{\mathrm{int}}$

with the admisible set $\mathcal{U}_{\mathrm{ad}}^{\mathrm{int}}=\mathcal{U}_{\mathrm{ad}}\left(u_{\mathrm{a}}^{\mathrm{i}}, u_{\mathrm{b}}^{\mathrm{i}}\right) \cap \mathcal{U}^{\mathrm{int}}$.
Theorem 1 Problem $(\hat{\mathbf{P}})$ admits a unique solution $\bar{u} \in \mathcal{U}_{\mathrm{ad}}^{\mathrm{int}}$.

Proof First of all, let us observe that

$\mathcal{U}_{\mathrm{ad}}^{\mathrm{int}}=\left\{\left(u^{\mathrm{c}}, u^{\mathrm{i}}\right) \in \mathcal{U} \mid u^{\mathrm{c}} \in\left[u_{\mathrm{a}}^{\mathrm{c}}, u_{\mathrm{b}}^{\mathrm{c}}\right], u^{\mathrm{i}} \in\left[u_{\mathrm{a}}^{\mathrm{i}}, u_{\mathrm{b}}^{\mathrm{i}}\right] \cap \mathbb{Z}^{N^{\mathrm{i}}}\right\}$

where $u^{\mathrm{c}} \in\left[u_{\mathrm{a}}^{\mathrm{c}}, u_{\mathrm{b}}^{\mathrm{c}}\right]$ stands for

$u_{\mathrm{a}}^{\mathrm{c}}(t) \leq u^{\mathrm{c}}(t) \leq u_{\mathrm{b}}^{\mathrm{c}}(t)$ in $\mathbb{R}^{N^{\mathrm{c}}}$ for almost all $t \in[0, T]$

and the set $\left[u_{\mathrm{a}}^{\mathrm{i}}, u_{\mathrm{b}}^{\mathrm{i}}\right] \cap \mathbb{Z}^{N^{\mathrm{i}}}$ is obviously finite. It therefore follows directly that

$$
\begin{aligned}
& \min _{u \in \mathcal{U}_{\mathrm{ad}}^{\mathrm{int}}\left(u_{\mathrm{a}}^{\mathrm{i}}, u_{\mathrm{b}}^{\mathrm{i}}\right)} \hat{J}(u)= \\
& \min _{u^{\mathrm{i}} \in\left[u_{\mathrm{a}}^{\mathrm{i}}, u_{\mathrm{b}}^{\mathrm{i}}\right] \cap \mathbb{Z}^{N^{\mathrm{i}}}} \underbrace{\left[\min _{u^{\mathrm{c}} \in\left[u_{\mathrm{a}}^{\mathrm{c}}, u_{\mathrm{b}}^{\mathrm{c}}\right]}\left\{\hat{J}(u) \mid u=\left(u^{\mathrm{c}}, u^{\mathrm{i}}\right)\right\}\right]}_{=:\left(\hat{\mathbf{Q}}_{u^{\mathrm{i}}}\right)}
\end{aligned}
$$

Here, $\left(\hat{\mathbf{Q}}_{u^{\mathrm{i}}}\right)$ is a problem in which the boundary variable $u^{\mathrm{i}}$ is fixed and only the distributed variable $u^{c}$ may vary. It is then well-known [28] that each linear-quadratic subproblem $\left(\hat{\mathbf{Q}}_{u^{\mathrm{i}}}\right)$ admits a unique minimizer $\bar{u}^{\mathrm{c}}\left(u^{\mathrm{i}}\right) \in\left[u_{\mathrm{a}}^{\mathrm{c}}, u_{\mathrm{b}}^{\mathrm{c}}\right]$ such that $(\hat{\mathbf{P}})$ has the unique solution

$\bar{u}=\arg \min \left\{\hat{J}\left(\bar{u}^{\mathrm{c}}\left(u^{\mathrm{i}}\right), u^{\mathrm{i}}\right) \mid u^{\mathrm{i}} \in\left[u_{\mathrm{a}}^{\mathrm{i}}, u_{\mathrm{b}}^{\mathrm{i}}\right] \cap \mathbb{Z}^{N^{\mathrm{i}}}\right\}$

Note that the proof of Theorem 1 could be utilized for the numerical solution of $(\hat{\mathbf{P}})$ : By iterating over all possible values $u^{\mathrm{i}} \in\left[u_{\mathrm{a}}^{\mathrm{i}}, u_{\mathrm{b}}^{\mathrm{i}}\right] \cap \mathbb{Z}^{N^{\mathrm{i}}}$, we could solve the problem $\left(\hat{\mathbf{Q}}_{u^{\mathrm{i}}}\right)$ and then pick the minimal function value. However, this is generally a bad idea since the computational effort grows very quickly with the cardinality of the set $\left[u_{\mathrm{a}}^{\mathrm{i}}, u_{\mathrm{b}}^{\mathrm{i}}\right] \cap \mathbb{Z}^{N^{\mathrm{i}}}$ and will not be acceptable but for the most simple of cases. In Section 6 we will present a better alternative.

\section{Galerkin discretization of the state equation}

System (3) represents a problem posed in infinite-dimensional function spaces. It is solved in practice by a discretization method. Given linearly independent spatial basis functions $\varphi_{1}, \ldots, \varphi_{m} \in V$, the space $V$ is replaced by an $m$-dimensional subspace $V^{h}=\operatorname{span}\left\{\varphi_{1}, \ldots, \varphi_{m}\right\}$. We endow $V^{h}$ with the $V$-topology. Since $V^{h}$ is of finite dimension, $V^{h}$ can be identified with $\left(V^{h}\right)^{\prime}$ so we have an isomorphism

$L^{2}\left(0, T ; V^{h}\right) \cap H^{1}\left(0, T,\left(V^{h}\right)^{\prime}\right) \cong H^{1}\left(0, T, V^{h}\right)$

A Galerkin method is employed to replace the infinite-dimensional problem (3) by a finite-dimensional version for almost all $t \in(0, T)$. Typically, $m$ is a very large number. Thus, we refer to the solution of the resulting system as a high-fidelity solution: A function $y^{h} \in H^{1}\left(0, T ; V^{h}\right)$ is called a high-fidelity solution to (3) if it holds for every $\varphi^{h} \in V^{h}$ 


$$
\begin{aligned}
& \left\langle y_{t}^{h}(t), \varphi^{h}\right\rangle_{V^{\prime} \times V}+\kappa \int_{\Omega} \nabla y^{h}(t, x) \cdot \nabla \varphi^{h}(x) \mathrm{d} x= \\
& \sum_{j=1}^{N^{\mathrm{c}}} u_{j}^{\mathrm{c}}(t) \int_{\Omega} \chi_{j}^{\mathrm{c}}(x) \varphi^{h}(x) \mathrm{d} x+\sum_{j=1}^{N^{\mathrm{i}}} u_{j}^{\mathrm{i}} \int_{\Gamma} \chi_{j}^{\mathrm{i}}(t, s) \varphi^{h}(s) \mathrm{d} s
\end{aligned}
$$

as well as

$y^{h}(0)=\mathcal{P}^{h} y_{\circ}$

where $\mathcal{P}^{h}: H \rightarrow V^{h}$ is a linear projection operator.

Example 1 Let us present a possible choice for the operator $\mathcal{P}^{h}$. For any $w \in H$ the element $v^{h}=\mathcal{P}^{h} w \in V^{h}$ is given as

$$
\left\langle v^{h}, \varphi_{i}^{h}\right\rangle_{H}=\left\langle w, \varphi_{i}^{h}\right\rangle_{H} \quad(i=1, \ldots, m)
$$

Utilizing the representation $v^{h}=\sum_{j=1}^{m} \mathrm{v}_{j}^{h} \varphi_{j}^{h} \in V^{h}$ the coefficient vector $\mathrm{v}^{h}=\left(\mathrm{v}_{1}^{h}, \ldots, \mathrm{v}_{m}^{h}\right)^{\top} \in \mathbb{R}^{m}$ is uniquely determined as the solution to the linear system

$\mathbf{M}^{h} \mathbf{v}^{h}=\mathrm{w}^{h}$

with the mass matrix $\mathrm{M}^{h}=\left(\left(\left\langle\varphi_{j}^{h}, \varphi_{i}^{h}\right\rangle_{H}\right)\right) \in \mathbb{R}^{m \times m}$ and the right-hand side $\mathrm{w}^{h}=\left(\left\langle w, \varphi_{1}^{h}\right\rangle_{H}, \ldots,\left\langle w, \varphi_{m}^{h}\right\rangle_{H}\right)^{\top} \in \mathbb{R}^{m}$.

It follows by the same arguments as for (3) that there exists a unique solution $y^{h} \in H^{1}\left(0, T ; V^{h}\right)$ to (4). In addition, there is an affine-linear, continuous control-to-state operator $\mathcal{S}^{h}: \mathcal{U} \rightarrow \mathcal{Y}$, where $y^{h}=\mathcal{S}^{h} u$ solves (4) for $u \in \mathcal{U}$. Again, we introduce the linear and bounded operator $\mathcal{S}_{0}^{h}$ : $\mathcal{U} \rightarrow H^{1}\left(0, T ; V^{h}\right) \subset \mathcal{y}$ which maps a control $u \in \mathcal{U}$ to the solution of (4) with $y_{\circ}=0$ and $\hat{y}^{h} \in H^{1}\left(0, T ; V^{h}\right)$ is the solution to (4) with $u=0$.

In the numerical realization $(\hat{\mathbf{P}})$ is replaced by the following discretized minimization problem

$\min \hat{J}^{h}(u):=J\left(\mathcal{S}^{h} u, u\right) \quad$ s.t. $\quad u \in \mathcal{U}_{\mathrm{ad}}^{\mathrm{int}}$

To solve $\left(\hat{\mathbf{P}}^{h}\right)$ we apply the branch-and-bound method; cf. Section 6 . This requires to solve many relaxed optimal control problems of the following type

$\min \hat{J}^{h}(u) \quad$ s.t. $\quad u \in \mathcal{U}_{\mathrm{ad}}(\mathrm{a}, \mathrm{b})$

where no integrality condition is present and the vectors a, b belong to $\left[u_{\mathrm{a}}^{\mathrm{i}}, u_{\mathrm{b}}^{\mathrm{i}}\right] \subset \mathbb{R}^{N^{\mathrm{i}}}$. It follows that the reduced cost $\hat{J}^{h}$ - considered as a function on $\mathcal{U}$ - is continuously Fréchet differentiable; cf. [28]. Its gradient $\nabla \hat{J}^{h}(u)$ at a point $u=$ $\left(u^{\mathrm{C}}, u^{\mathrm{i}}\right) \in \mathcal{U}$ is given as

$\nabla \hat{J}^{h}(u)=\left(\frac{\nabla^{\mathrm{c}} \hat{J}^{h}(u)}{\nabla^{\mathrm{i}} \hat{J}^{h}(u)}\right) \in \mathcal{U}$

In (5) we use the notation

$\nabla^{\mathrm{c}} \hat{J}^{h}(u)=\left(\begin{array}{c}\alpha_{1}^{\mathrm{c}} u_{1}^{\mathrm{c}}(\cdot)+\int_{\Omega} \chi_{1}^{\mathrm{c}}(x) p^{h}(\cdot, x) \mathrm{d} x \\ \vdots \\ \alpha_{N^{\mathrm{c}}}^{\mathrm{c}} u_{N^{\mathrm{c}}}^{\mathrm{c}}(\cdot)+\int_{\Omega} \chi_{N^{\mathrm{c}}}^{\mathrm{c}}(x) p^{h}(\cdot, x) \mathrm{d} x\end{array}\right)$ and

$\nabla^{\mathrm{i}} \hat{J}^{h}(u)=\left(\begin{array}{c}\alpha_{1}^{\mathrm{i}}\left(u_{1}^{\mathrm{i}}-\hat{u}_{1}^{\mathrm{i}}\right)+\int_{0}^{T} \int_{\Gamma} \chi_{1}^{\mathrm{i}}(x) p^{h}(t, x) \mathrm{d} x \mathrm{~d} t \\ \vdots \\ \alpha_{N^{\mathrm{i}}}^{\mathrm{i}}\left(u_{N^{\mathrm{i}}}^{\mathrm{i}}-\hat{u}_{N^{\mathrm{i}}}^{\mathrm{i}}\right)+\int_{0}^{T} \int_{\Gamma} \chi_{N^{\mathrm{i}}}^{\mathrm{i}}(x) p^{h}(t, x) \mathrm{d} x \mathrm{~d} t\end{array}\right)$

where the dual variable $p^{h} \in H^{1}\left(0, T ; V^{h}\right)$ satisfies the dual or adjoint problem

$-\left\langle p_{t}^{h}(t), \varphi^{h}\right\rangle_{V^{\prime} \times V}+\kappa \int_{\Omega} \nabla p^{h}(t, x) \cdot \nabla \varphi^{h}(x) \mathrm{d} x=$
$\int_{\Omega} \alpha^{Q}\left(y^{h}(t, x)-y_{d}(t, x)\right) \varphi^{h}(x) \mathrm{d} x \quad \forall \varphi^{h} \in V^{h}$

as well as

$p^{h}(T)=0$

with $y^{h}=\mathcal{S}^{h} u$. Using similar arguments as for (3) it can be shown that (6) has a unique solution $p^{h}$ for any $u \in \mathcal{U}$. Another way to write the gradient is by using the solution operators $\mathcal{S}^{h}$ and $\mathcal{S}_{0}^{h}$. In particular, we have the structure

$\nabla \hat{J}^{h}(u)=\left(\begin{array}{c}\alpha_{1}^{\mathrm{c}} u_{1}^{\mathrm{c}}(\cdot) \\ \vdots \\ \frac{\alpha_{N^{\mathrm{c}}}^{\mathrm{c}} u_{N^{\mathrm{c}}}^{\mathrm{c}}(\cdot)}{\alpha_{1}^{\mathrm{i}}\left(u_{1}^{\mathrm{i}}-\hat{u}_{1}^{\mathrm{i}}\right)} \\ \vdots \\ \alpha_{N^{\mathrm{i}}}^{\mathrm{i}}\left(u_{N^{\mathrm{i}}}^{\mathrm{i}}-\hat{u}_{N^{\mathrm{i}}}^{\mathrm{i}}\right)\end{array}\right)+\alpha^{Q} \mathcal{S}_{0}^{h *}\left(\mathcal{S}^{h} u-y_{d}\right)$

where $\mathcal{S}_{0}^{h *}: W(0, T) \rightarrow \mathcal{U}$ is the adjoint operator of $\mathcal{S}_{0}^{h}: \mathcal{U} \rightarrow$ $W(0, T)$. From (7) we can immediately see that $\hat{J}^{h}$ is even twice Fréchet differentiable with constant second derivative

$\left(\nabla^{2} \hat{J}^{h}\right) v=\left(\begin{array}{c}\alpha_{1}^{\mathrm{c}} v_{1}^{\mathrm{c}}(\cdot) \\ \vdots \\ \alpha_{N^{\mathrm{c}}}^{\mathrm{c}} v_{N^{\mathrm{c}}}^{\mathrm{c}}(\cdot) \\ \alpha_{1}^{\mathrm{i}} v_{1}^{\mathrm{i}} \\ \vdots \\ \alpha_{N^{\mathrm{i}}}^{\mathrm{i}} v_{N^{\mathrm{i}}}^{\mathrm{i}}\end{array}\right)+\alpha^{Q} \mathcal{S}_{0}^{h *} \mathcal{S}_{0}^{h} v$

From (8) it follows directly that $\nabla^{2} \hat{J}^{h}$ is uniformly coercive with

$\left\langle\left(\nabla^{2} \hat{J}^{h}\right) v, v\right\rangle u \geq \alpha\|v\|_{u}^{2}$

where

$\alpha=\min \left\{\alpha_{1}^{\mathrm{c}}, \ldots, \alpha_{N^{\mathrm{c}}}^{\mathrm{c}}, \alpha_{1}^{\mathrm{i}}, \ldots, \alpha_{N^{\mathrm{i}}}^{\mathrm{i}}\right\}>0$

Since $\left(\hat{\mathbf{P}}_{\mathrm{ab}}^{h}\right)$ is a linear-quadratic optimal control problem with bilateral constraints. Moreover, $\hat{J}^{h}$ is strictly convex and $\mathcal{U}_{\mathrm{ad}}(\mathrm{a}, \mathrm{b})$ is a convex set. Hence, there exists a unique 
minimizer $\bar{u}_{\mathrm{ab}} \in \mathcal{U}_{\mathrm{ad}}(\mathrm{a}, \mathrm{b})$. Further, $\bar{u}_{\mathrm{ab}}$ satisfies the firstorder sufficient optimality conditions

$\left\langle\nabla \hat{J}^{h}\left(\bar{u}_{\mathrm{ab}}\right), u-\bar{u}_{\mathrm{ab}}\right\rangle u \geq 0 \quad$ for all $u \in \mathcal{U}_{\mathrm{ad}}(\mathrm{a}, \mathrm{b})$

Suppose that $\widehat{u} \in \mathcal{U}_{\mathrm{ad}}(\mathrm{a}, \mathrm{b})$ be an arbitrarily admissible control with $\widehat{u} \neq \bar{u}_{\mathrm{ab}}$. Then, $\widehat{u}$ does not satisfy (10). However, there exists a perturbation function $\xi=\left(\xi^{\mathrm{c}}, \xi^{\mathrm{i}}\right) \in \mathcal{U}$ (cf. [21] and $[1,5])$ such that

$\left\langle\nabla \hat{J}^{h}(\widehat{u})+\xi, u-\widehat{u}\right\rangle_{u} \geq 0 \quad$ for all $u \in \mathcal{U}_{\mathrm{ad}}(\mathrm{a}, \mathrm{b})$

The function $\xi$ can be computed as stated in the next theorem.

Theorem 2 Let $\widehat{u}=\left(\widehat{u}^{\mathrm{c}}, \widehat{u}^{\mathrm{i}}\right) \in \mathcal{U}_{\mathrm{ad}}(\mathrm{a}, \mathrm{b})$ be an arbitrary control satisfying $\widehat{u} \neq \bar{u}_{\mathrm{ab}}$. We define $\xi(\widehat{u})=\left(\xi^{\mathrm{C}}(\widehat{u}), \xi^{\mathrm{i}}(\widehat{u})\right) \in \mathcal{U}$ by

$$
\begin{aligned}
& \xi_{j}^{\mathrm{c}}(\widehat{u})(t) \\
& \quad= \begin{cases}\max \left\{0,-\left(\nabla^{\mathrm{c}} \hat{J}^{h}(\widehat{u})\right)_{j}(t)\right\} & \text { if } \widehat{u}_{j}^{\mathrm{c}}(t)=\left(u_{\mathrm{a}}^{\mathrm{c}}\right)_{j}(t) \\
\min \left\{0,-\left(\nabla^{\mathrm{c}} \hat{J}^{h}(\widehat{u})\right)_{j}(t)\right\} & \text { if } \widehat{u}_{j}^{\mathrm{c}}(t)=\left(u_{\mathrm{b}}^{\mathrm{c}}\right)_{j}(t) \\
-\left(\nabla^{\mathrm{c}} \hat{J}^{h}(\widehat{u})\right)_{j}(t) & \text { otherwise }\end{cases}
\end{aligned}
$$

for $j=1, \ldots, N^{\mathrm{c}}$ and

$\xi_{j}^{\mathrm{i}}(\widehat{u})= \begin{cases}0 & \text { if } \mathrm{a}_{j}=\mathrm{b}_{j} \\ \max \left\{0,-\left(\nabla^{\mathrm{i}} \hat{J}^{h}(\widehat{u})\right)_{j}\right\} & \text { if } \widehat{\mathrm{u}}_{j}^{\mathrm{i}}=\mathrm{a}_{j} \\ \min \left\{0,-\left(\nabla^{\mathrm{i}} \hat{J}^{h}(\widehat{u})_{j}\right\}\right. & \text { if } \widehat{u}_{j}^{\mathrm{i}}=\mathrm{b}_{j} \\ -\left(\nabla^{\mathrm{i}} \hat{J}^{h}(\widehat{u})_{j}\right. & \text { otherwise }\end{cases}$

for $j=1, \ldots, N^{\mathrm{i}}$. Then, $\xi(\widehat{u})$ satisfies $(11)$ and the a posteriori error estimator holds

$\left\|\widehat{u}-\bar{u}_{\mathrm{ab}}\right\|_{u} \leq \Delta_{u}(\widehat{u})=\frac{1}{\bar{\alpha}}\|\xi(\widehat{u})\|_{u}$

with the coercivity constant $\bar{\alpha}$ from (9).

Proof First of all, it holds for every $v \in \mathcal{U}$ :

$$
\begin{aligned}
& \left\langle\nabla \hat{J}^{h}(\widehat{u})+\xi(\widehat{u}), v-\widehat{u}\right\rangle_{u} \\
= & \sum_{i=1}^{N^{\mathrm{c}}} \int_{0}^{T}\left(\nabla^{\mathrm{c}} \hat{J}^{h}(\widehat{u})(t)+\xi^{\mathrm{c}}(\widehat{u})(t)\right)_{i}\left(v_{i}^{\mathrm{c}}(t)-u_{i}^{\mathrm{c}}(t)\right) \mathrm{d} t \\
& +\sum_{j=1}^{N^{\mathrm{i}}}\left(\nabla^{\mathrm{i}} \hat{J}^{h}(u)+\xi^{\mathrm{i}}(u)\right)_{j}\left(v_{j}^{\mathrm{i}}-u_{j}^{\mathrm{i}}\right) \geq 0
\end{aligned}
$$

directly from Definition (12). For more details we refer the reader to [29]. By especially choosing $v=\bar{u}$ and adding it to (10), we obtain

$$
\begin{aligned}
0 & \leq\left\langle\nabla \hat{J}^{h}(\bar{u}), \widehat{u}-\bar{u}\right\rangle_{\mathcal{U}}+\left\langle\nabla \hat{J}^{h}(\widehat{u})+\xi(\widehat{u}), \bar{u}-\widehat{u}\right\rangle_{\mathcal{U}} \\
& =-\left\langle\nabla \hat{J}^{h}(\widehat{u})-\nabla \hat{J}^{h}(\bar{u}), \widehat{u}-\bar{u}\right\rangle_{\mathcal{U}}-\langle\xi(\widehat{u}), \widehat{u}-\bar{u}\rangle_{U}
\end{aligned}
$$

Now we utilize the fact that $\hat{J}^{h}$ is twice Fréchet differentiable and apply the mean value theorem, resulting in

$$
\begin{aligned}
0 & \leq-\left\langle\left(\nabla^{2} \hat{J}\right)(\widehat{u}-\bar{u}), \widehat{u}-\bar{u}\right\rangle+\left|\langle\xi(\widehat{u}), \widehat{u}-\bar{u}\rangle_{u}\right| \\
& \leq-\bar{\alpha}\|u-\bar{u}\|_{u}^{2}+\|\xi(\widehat{u})\|_{u}\|\widehat{u}-\bar{u}\|_{u}
\end{aligned}
$$

Dividing by $\|\widehat{u}-\bar{u}\|_{u}>0$ and rearranging the inequality results in (13) we conclude the proof.

\section{Reduced-order modeling}

To solve $\left(\hat{\mathbf{P}}_{\mathrm{ab}}^{h}\right)$ many realizations of the operator $\mathcal{S}^{h}$ are necessary. For this reason we make use of a reduced-order approach, where we replace (4) by a low-dimensional reducedorder model (ROM). In this work we utilize the proper orthogonal decomposition (POD) method to compute the ROMs; cf. [18].

\subsection{The POD method}

Assume that we have chosen a control $u \in \mathcal{U}_{\mathrm{ad}}(\mathrm{a}, \mathrm{b})$ and we would like to build a localized surrogate model which is highly accurate for the data associated with this control. Let $y^{h}=\mathcal{S}^{h} u$ and $p^{h}$ denote the associated solutions to (4) and (6), respectively. Then we consider the linear space of snapshots

$\mathcal{V}^{h}=\operatorname{span}\left\{y^{h}(t), p^{h}(t) \mid t \in[0, T]\right\} \subset V^{h} \subset V$

with $\mathrm{d}=\operatorname{dim} \nu^{h} \leq m$.

Remark 1 Note that not only the state but also the adjoint is included in the snapshot set. This is motivated by convergence results stated in $[13,17]$, for example. Moreover, time derivatives are not added to the snapshot set thanks to the a-priori error analysis carried out in [25] and [13].

For any finite $\ell \leq \mathrm{d}$ we are interested in determining a POD basis of rank $\ell$ which minimizes the mean square error between $y^{h}(t), p^{h}(t)$ and their corresponding $\ell$-th partial Fourier sums in the resulting subspace on average in $[0, T]$ :

$$
\left\{\begin{array}{l}
\min \int_{0}^{T}\left\|y^{h}(t)-\sum_{i=1}^{\ell}\left\langle y^{h}(t), \psi_{i}^{h}\right\rangle_{V} \psi_{i}^{h}\right\|_{V}^{2} \mathrm{~d} t \\
\quad+\int_{0}^{T}\left\|p^{h}(t)-\sum_{i=1}^{\ell}\left\langle p^{h}(t), \psi_{i}^{h}\right\rangle_{V} \psi_{i}^{h}\right\|_{V}^{2} \mathrm{~d} t \\
\text { s.t. }\left\{\psi_{i}^{h}\right\}_{i=1}^{\ell} \subset V^{h},\left\langle\psi_{i}^{h}, \psi_{j}^{h}\right\rangle_{V}=\delta_{i j}(1 \leq i, j \leq \ell)
\end{array}\right.
$$

A solution $\left\{\psi_{i}^{h}\right\}_{i=1}^{\ell}$ to $\left(\mathbf{P}^{\ell}\right)$ is called POD basis of rank $\ell$. Let us introduce the linear, compact, selfadjoint and nonnegative operator $\mathcal{R}^{h}: V \rightarrow V^{h}$ by

$\mathcal{R}^{h} \psi=\int_{0}^{T}\left\langle y^{h}(t), \psi\right\rangle_{V} y^{h}(t)+\left\langle p^{h}(t), \psi\right\rangle_{V} p^{h}(t) \mathrm{d} t$

Then, it is well-known (cf. [13]) that a solution $\left\{\psi_{i}^{h}\right\}_{i=1}^{\ell}$ to $\left(\mathbf{P}^{\ell}\right)$ is given by the eigenvectors associated with the $\ell$ largest eigenvalues of $\mathcal{R}^{h}$ :

$\mathcal{R}^{h} \psi_{i}^{h}=\lambda_{i}^{h} \psi_{i}^{h}(1 \leq i \leq \ell), \lambda_{1}^{h} \geq \ldots \geq \lambda_{\ell}^{h} \geq \lambda_{\ell+1}^{h} \geq \ldots \geq 0$ 
Moreover, the POD basis $\left\{\psi_{i}^{h}\right\}_{i=1}^{\ell}$ of rank $\ell$ satisfies $\psi_{i}^{h} \in$ $V^{h}$ for $1 \leq i \leq \ell$ and

$$
\begin{aligned}
& \int_{0}^{T}\left\|y^{h}(t)-\sum_{i=1}^{\ell}\left\langle y^{h}(t), \psi_{i}^{h}\right\rangle_{V} \psi_{i}^{h}\right\|_{V}^{2} \mathrm{~d} t \\
& \quad+\int_{0}^{T}\left\|p^{h}(t)-\sum_{i=1}^{\ell}\left\langle p^{h}(t), \psi_{i}^{h}\right\rangle_{V} \psi_{i}^{h}\right\|_{V}^{2} \mathrm{~d} t=\sum_{i=\ell+1}^{\mathrm{d}} \lambda_{i}^{h}
\end{aligned}
$$

Remark 2 (Discrete POD method) It was already mentioned in Section 4 that in the numerical implementation, the space $V$ is replaced by the high-fidelity space $V^{h}$. Apart from that, the integral in $\left(\mathbf{P}^{\ell}\right)$ has to be approximated numerically. Let a time grid be given by $0=t_{1}<\ldots<t_{n}=T$ along with quadrature weights $\gamma_{1}, \ldots, \gamma_{n}>0$ satisfying $\sum_{j=1}^{n} \gamma_{j}=T$. Further, suppose that $y_{j}^{h n}$ and $p_{j}^{h n}$ are approximations of $y^{h}\left(t_{j}\right)$ and $p^{h}\left(t_{j}\right)$, respectively, at the time instance $t_{j}(j=1, \ldots, n)$. For instance, $y_{j}^{h n}$ and $p_{j}^{h n}$ are computed by applying an implicit Euler method to (4) and (6), respectively. Then, the discrete version of $\left(\mathbf{P}^{\ell}\right)$ takes the form

$$
\left\{\begin{array}{l}
\min \sum_{j=1}^{n} \gamma_{j}\left\|y_{j}^{h n}-\sum_{i=1}^{\ell}\left\langle y_{j}^{h n}, \psi_{i}^{h n}\right\rangle_{V} \psi_{i}^{h n}\right\|_{V}^{2} \\
\quad+\sum_{j=1}^{n} \gamma_{j}\left\|p_{j}^{h n}-\sum_{i=1}^{\ell}\left\langle p_{j}^{h n}, \psi_{i}^{h n}\right\rangle_{V} \psi_{i}^{h n}\right\|_{V}^{2} \\
\text { s.t. }\left\{\psi_{i}^{h n}\right\}_{i=1}^{\ell} \subset V^{h},\left\langle\psi_{i}^{h n}, \psi_{j}^{h n}\right\rangle_{V}=\delta_{i j}(1 \leq i, j \leq \ell)
\end{array}\right.
$$

Analogously to the continuous problem $\left(\mathbf{P}^{\ell}\right)$, a solution to (14) is given by the eigenvectors to the $\ell$ largest eigenvalues of the operator $\mathcal{R}^{h n}: V \rightarrow V^{h}$ by

$\mathcal{R}^{h n} \psi=\sum_{j=1}^{n} \gamma_{j}\left(\left\langle y_{j}^{h n}, \psi\right\rangle_{V} y_{j}^{h n}+\left\langle p_{j}^{h n}, \psi\right\rangle_{V} p_{j}^{h n}\right)$

which is an approximation of $\mathcal{R}^{h}$.

\subsection{The POD Galerkin scheme}

Now let us suppose that we have computed a POD basis $\left\{\psi_{i}^{h}\right\}_{i=1}^{\ell} \subset V^{h}$ of rank $\ell$. We define the finite dimensional subspace

$V^{h \ell}=\operatorname{span}\left\{\psi_{1}^{h}, \ldots, \psi_{\ell}^{h}\right\} \subset V^{h}$

Then the POD solution operator $\mathcal{S}^{h \ell}: \mathcal{U} \rightarrow H^{1}\left(0, T ; V^{h \ell}\right)$ is defined as follows: $y^{h \ell}=\mathcal{S}^{h \ell} u$ with $y^{h \ell}(t) \in V^{h \ell}$ solves for almost all $t \in[0, T]$ the POD Galerkin scheme

$$
\begin{gathered}
\left\langle y_{t}^{h \ell}(t), \psi^{h}\right\rangle_{V^{\prime} \times V}+\kappa \int_{\Omega} \nabla y^{h \ell}(t, x) \cdot \nabla \psi^{h}(x) \mathrm{d} x \\
=\sum_{j=1}^{N^{c}} u_{j}^{\mathrm{c}}(t) \int_{\Omega} \chi_{j}^{\mathrm{c}}(x) \psi^{h}(x) \mathrm{d} x \\
\quad+\sum_{j=1}^{N^{\mathrm{i}}} u_{j}^{\mathrm{i}} \int_{\Gamma} \chi_{j}^{\mathrm{i}}(t, s) \psi^{h}(s) \mathrm{d} s
\end{gathered}
$$

for all $\psi^{h} \in V^{h \ell}$ as well as

$y^{h \ell}(0)=\mathcal{P}^{h \ell} y_{\circ}$

In (15a) we have used the following linear, $H$-orthogonal projection $\mathcal{P}^{h \ell}: H \rightarrow V^{h \ell}$ is given as:

$\mathcal{P}^{h \ell} \varphi$ minimizes $\inf _{\varphi^{h \ell} \in V^{h \ell}}\left\|\varphi-\varphi^{h \ell}\right\|_{H}$ for given $\varphi \in H$

Note that for every $\varphi \in V$ the projection $\mathcal{P}^{h \ell} \varphi \in V^{h \ell}$ is given as

$\mathcal{P}^{h \ell} \varphi=\sum_{i=1}^{\ell} c_{i} \psi_{i}^{h}$

where the coefficients $c_{i}, i=1, \ldots, \ell$, solve the linear system

$\sum_{j=1}^{\ell}\left\langle\psi_{j}^{h}, \psi_{i}^{h}\right\rangle_{H} c_{j}=\left\langle\varphi, \psi_{i}^{h}\right\rangle_{H} \quad$ for $i=1, \ldots, \ell$

The adjoint equation (6) is also reduced in a similar way; cf. [13]. Now, $\left(\hat{\mathbf{P}}_{\mathrm{ab}}^{h}\right)$ is replaced by its POD Galerkin approximation. We consider the optimal control problem

$\min \hat{J}^{h \ell}(u):=J\left(\mathcal{S}^{h \ell} u, u\right) \quad$ s.t. $\quad u \in \mathcal{U}_{\mathrm{ad}}(\mathrm{a}, \mathrm{b})$

$\left(\hat{\mathbf{P}}_{\mathrm{ab}}^{h \ell}\right)$

with vectors $\mathrm{a}, \mathrm{b} \in\left[u_{\mathrm{a}}^{\mathrm{i}}, u_{\mathrm{b}}^{\mathrm{i}}\right] \subset \mathbb{R}^{N^{\mathrm{i}}}$ satisfying $\mathrm{a} \leq \mathrm{b}$.

Remark 3 Thanks to the reduced-order approach, $\left(\hat{\mathbf{P}}_{a b}^{h \ell}\right)$ can be solved computationally much faster than $\left(\hat{\mathbf{P}}_{\mathrm{ab}}^{h}\right)$. Here, we can rely on existing techniques for linear-quadratic problems; cf. [13]. In our numerical experiments we solve $\left(\hat{\mathbf{P}}_{\mathrm{ab}}^{h}\right)$ only for the root node of the branch-and-bound method, i.e., $\mathrm{a}=u_{\mathrm{a}}^{\mathrm{i}}$ and $\mathrm{b}=u_{\mathrm{b}}^{\mathrm{i}}$. The obtained optimal state and adjoint are utilized as snaphots in $V^{h}$ for the computation of the POD basis of rank $\ell$. Then, the reduced-order approximation $\hat{J}^{h \ell}$ of $\hat{J}^{h}$ is computed and in the following nodes of the branchand-bound method we solve $\left(\hat{\mathbf{P}}_{\mathrm{ab}}^{h \ell}\right)$ to get the associated optimal control $\bar{u}_{\mathrm{ab}}^{\ell} \in \mathcal{U}_{\mathrm{ad}}(\mathrm{a}, \mathrm{b})$ with $\mathrm{a}, \mathrm{b} \in\left[u_{\mathrm{a}}^{\mathrm{i}}, u_{\mathrm{b}}^{\mathrm{i}}\right]$ satisfying $\mathrm{a} \leq \mathrm{b}$. Then, by setting $\widehat{u}=\bar{u}_{\mathrm{ab}}^{\ell}$ the error between $\bar{u}_{\mathrm{ab}}^{\ell}$ and $\bar{u}_{\mathrm{ab}}$ can be estimated by the a posteriori error presented in Theorem 2. Moreover, the a priori error in the root node can be estimated as [13]:

$\left\|\bar{u}_{\mathrm{ab}}^{\ell}-\bar{u}_{\mathrm{ab}}\right\|_{\mathrm{u}}^{2} \leq C \sum_{i=\ell+1}^{\mathrm{d}} \lambda_{i}^{h}\left\|\psi_{i}^{h}-\mathcal{P}^{h \ell} \psi_{i}^{h}\right\|_{V}^{2}$

If the error is too large, we increase the number $\ell$ of POD basis functions. 


\section{Branch-and-Bound method}

Our model requires that all the boundary controls $u^{\mathrm{i}}$ are integer, while the distributed controls $u^{c}$ are allowed to be continuous within given bilateral control constraints. In order to deal with the mixed-integer, time-dependent constraints, a branch-and-bound algorithm was implemented (see Algorithm 1). The idea is based on solving a set of correlated relaxation problems by omitting the integrality conditions and computing numerically the following type of problem:

$\min \hat{\jmath}(u) \quad$ s.t. $\quad u \in \mathcal{U}_{\mathrm{ad}}(\mathrm{a}, \mathrm{b}) \quad\left(\hat{\mathbf{P}}_{\mathrm{ab}}\right)$

For $\left(\hat{\mathbf{P}}_{\mathrm{ab}}^{h}\right)$ we have $\hat{\mathrm{J}}=\hat{J}^{h}$ and for $\left(\hat{\mathbf{P}}_{\mathrm{ab}}^{h \ell}\right)$ we take $\hat{\mathrm{J}}=\hat{J}^{h \ell}$.

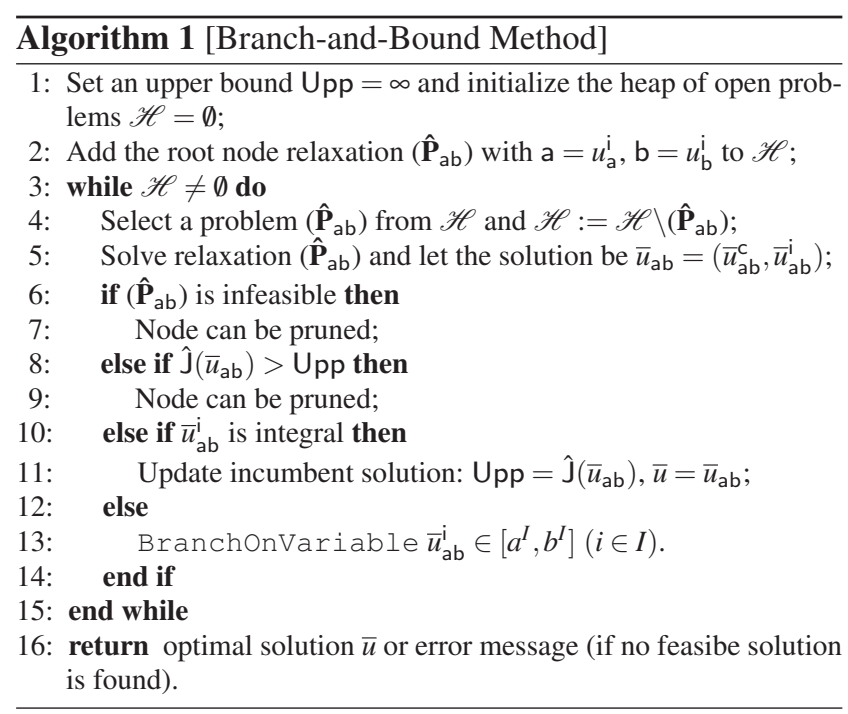

Suppose that $\bar{u}_{\mathrm{ab}}=\left(\bar{u}_{\mathrm{ab}}^{\mathrm{c}}, \bar{u}_{\mathrm{ab}}^{\mathrm{i}}\right) \in \mathcal{U}_{\mathrm{ad}}(\mathrm{a}, \mathrm{b})$ is a solution to $\left(\hat{\mathbf{P}}_{\mathrm{ab}}\right)$. If the $j$-th component $\bar{u}_{\mathrm{ab}, j}^{\mathrm{i}}\left(j \in\left\{1, \ldots, N^{\mathrm{i}}\right\}\right)$ of $\bar{u}_{\mathrm{ab}}^{\mathrm{i}} \in$ $\mathbb{R}^{N^{\mathrm{i}}}$ is not integer, we branch on the variable $\bar{u}_{\mathrm{ab}}^{\mathrm{i}}$ as follows: Set

$\tilde{\mathrm{a}}_{j}:=\left\lfloor\bar{u}_{\mathrm{ab}, j}^{\mathrm{i}}\right\rfloor \geq u_{\mathrm{a}, j}^{\mathrm{i}}, \quad \tilde{\mathrm{b}}=\left(\mathrm{a}_{1}, \ldots, \mathrm{a}_{j-1}, \tilde{\mathrm{a}}_{j}, \mathrm{a}_{j+1}, \ldots, \mathrm{a}_{N^{\mathrm{i}}}\right)$

$\tilde{\mathrm{b}}_{j}:=\left\lceil\bar{u}_{\mathrm{ab}, \mathrm{j}}^{\mathrm{i}}\right\rceil \leq u_{\mathrm{b}, j}^{\mathrm{i}}, \quad \tilde{\mathrm{a}}=\left(\mathrm{b}_{1}, \ldots, \mathrm{b}_{j-1}, \tilde{\mathrm{b}}_{j}, \mathrm{~b}_{j+1}, \ldots, \mathrm{b}_{N^{\mathrm{i}}}\right)$

Then, add the corresponding subproblems $\left(\hat{\mathbf{P}}_{\mathrm{ab}}\right)$ and $\left(\hat{\mathbf{P}}_{\tilde{a} \mathrm{~b}}\right)$ to $\mathscr{H}$.

Since the relaxation problem can always be solved to its optimality and the set of integer boundary controls is finite, it follows immediately that the branch-and-bound algorithm terminates at an optimal solution after solving a finite number of nodes or with a certificate that the problem $\left(\hat{\mathbf{P}}_{\mathrm{ab}}\right)$ has no solution.

\subsection{Branching Strategies}

We choose the strong branching strategy for computing our test cases. Let $I_{c} \subset\left\{1, \ldots, N^{\mathrm{i}}\right\}$ denote the set of all candidates of variables to be branched on. By branching, we are interested in increasing the lower bound of our problem as much as possible in order to reduce the size of the branchand-bound tree. The idea of strong branching is to solve all the possible child subproblems each time when a branching is necessary. Suppose that we branch on the variable $u_{i}$ $\left(i \in I_{c}\right)$ and let $\hat{\mathrm{j}}_{i}^{+}$and $\hat{\mathrm{J}}_{i}^{-}$be the optimal objective values of the child subproblems. We compute $D_{i}^{-}$and $D_{i}^{+}$by

$D_{i}^{-}=\hat{\mathrm{J}}_{i}^{-}-\hat{\mathrm{J}}_{i}$ and $D_{i}^{+}=\hat{\mathrm{J}}_{i}^{+}-\hat{\mathrm{J}}_{i}$,

where $\hat{\mathrm{J}}_{i}$ denotes the optimal objective value at the parent node. We then choose the branching candidate $i \in I_{C}$ which maximizes the so-called score function

$s_{i}:=\mu \min \left(D_{i}^{-}, D_{i}^{+}\right)+(1-\mu) \max \left(D_{i}^{-}, D_{i}^{+}\right)$,

where $\mu \in[0,1]$ is a fixed parameter.

Strong branching is particularly effective in reducing the number of nodes in the branch-and-bound searching tree, but the computational burden of solving each added subproblem could be significantly large. Practically, it may be more efficient to solve the subproblems not exactly but only approximately. Or even better, one could also estimate the measure $D_{i}^{ \pm}$based on some a priori information and do not need to solve the subproblems at all for maximizing the score function. Nevertheless, as we will see later, strong branching performs sufficiently well for our test cases, since the computation at nodes is not too expensive and the size of branch-and-bound tree is relatively small.

\subsection{Node Selection}

We distinguish three strategies: depth-first search, best search and a combination of the two.

Depth-first search selects the deepest node in the tree, in our case this will be the last node added to the heap of open problems. An advantage of this strategy is that we have to store as few as possible open problems, but unfortunately if no upper bound is found it will solve many nodes with a lower bound that is actually larger than the solution, leading to a poor performance.

Best-bound search selects the node with the best lower bound. This strategy minimizes the number of explored nodes for a fixed sequence of branching decisions, as these would have been solved independently of the upper bound. On the other hand more open problems have to be stored and mostly an integer solution will not be found until the end of the search. Hence if the solution time is limited, it might not find any feasible point at all.

Two-phase method starts with the depth-first strategy until a feasible point for $\left(\hat{\mathbf{P}}_{\mathrm{ab}}\right)$ was found and then continues 


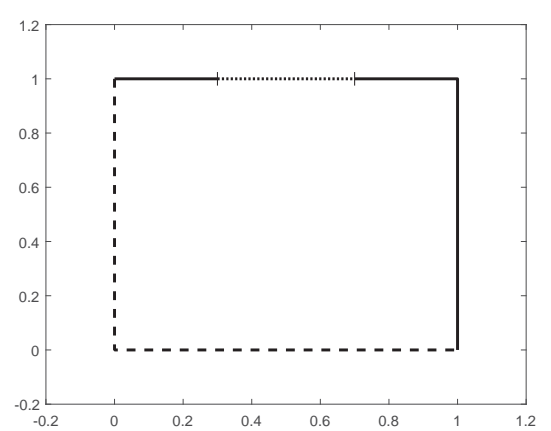

Fig. 1 The domain $\Omega$ with interior walls (dashed lines), outer walls (solid lines) and a window at $0.3 \leq x_{1} \leq 0.7, x_{2}=1$ (dotted line).

with the best-search strategy. We store very few information about each node only, so the search tree will stay manageable, but otherwise one could also think about switching back.

\section{Numerical experiments}

We will consider a squared room $\Omega:=(0,1)^{2}$ with two interior walls and two outer walls and a window in the middle of one of the latter as shown in Fig. 1. The boundary control $u^{\mathrm{i}} \in \mathbb{Z}^{3}$ corresponds to the choices of insulation material for the three exterior boundary segments: the long outer wall around the up-right corner $\Gamma_{1}$, the window $\Gamma_{2}$ and the short outer wall $\Gamma_{3}$. For the window the insulation material shall be more expensive and the heat transition will be increased. This is represented by

$\alpha_{1}^{\mathrm{i}}=0.1, \quad \alpha_{2}^{\mathrm{i}}=0.2, \quad \alpha_{3}^{\mathrm{i}}=0.1$

and

$\chi_{j}^{\mathrm{i}}(t, s)=-\alpha_{j}^{\mathrm{i}}\left(22-y_{a}(t)\right), \quad(t, s) \in \Sigma_{j}, \quad j=1,2,3$

The boundary control is restricted by $u_{\mathrm{a}}^{\mathrm{i}}=(1,2,1)$ from below as the window part cannot be isolated as well as the walls and $u_{\mathrm{b}}^{\mathrm{i}}=(10,10,10)$ from above. The distributed control $u^{\mathrm{c}}$ represents an underfloor heating, either one heating tile or four squared tiles in counterclockwise order starting from the lower-left corner. It is allowed to rank from 0 to 10 and can vary over time. Unless specified otherwise $\alpha^{\mathrm{c}}=0.3$ and $\alpha^{Q}=10$. The desired temperature will be $y_{d}=22^{\circ} \mathrm{C}$ and the initial inside temperature $y_{\circ}=18^{\circ} \mathrm{C}$.

The triangulation of $\Omega$ satisfies a maximal mesh size of 0.05 and consists of $m=721$ unknowns. All arising optimal control problems are solved with the projected Newton-CG method. As stated in Remark 3 we run a full optimization in the first node, from which we compute a POD basis of maximal rank $d$ with the MATLAB built-in eigenvalue decomposition. The number $\ell$ of POD basis elements is then

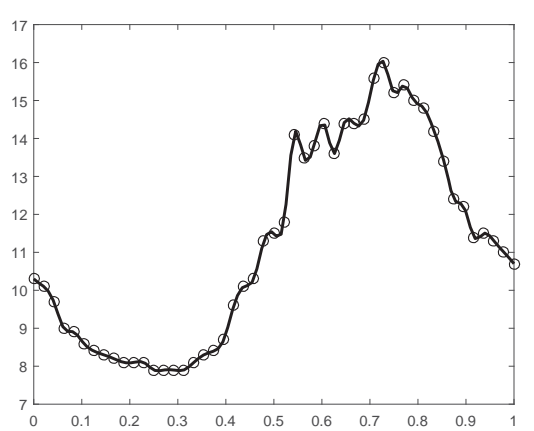

Fig. 2 Run 1: Set of temperature measurements (circles) from Konstanz from midnight till midnight on May 3rd and splinewise interpolated outside temperature $y_{a}$ (solid line).

chosen individually at each node such that for the energy holds

$\mathcal{E}(\ell)=\frac{\sum_{i=1}^{\ell} \lambda_{i}^{h}}{\sum_{i=1}^{\mathrm{d}} \lambda_{i}^{h}}>0.99999$

Recall that the error between the POD suboptimal control to the optimal one computed by the full optimization can be estimated in terms of $\mathcal{E}(\ell)$ provided the POD basis is derived from the full optimization; cf. [13]. If the a-posteriori estimate is larger than the tolerance 1 , then we enlargen the POD basis by a few basis vectors such that $\mathcal{E}(\ell)>0.9999999$. At each node the optimization will start from the canonic projection of the previously optimal (and after the branching infeasible) control onto the set of feasible controls. The variable to branch upon will be selected from the set of candidates according to the strong branching strategy. The node selection will be performed by the two-phase method.

The implementation is done by using MATLAB Release $2016 \mathrm{a}$ and all test runs are performed on a $2.5 \mathrm{GHz}$ Intel inside core i5 processor with 8 GB RAM.

\subsection{Weather data of Constance, Germany}

Throughout this subsection we will consider an outside temperature based on measurements in Konstanz on May 3rd as illustrated in Fig. $2^{1}$.

Run 1. We start with one underfloor heating tile acting on the whole domain $\Omega$. Solving this problem without model reduction, i.e. solving $\left(\hat{\mathbf{P}}^{h}\right)$, required 28.37 seconds and yields the same branching decisions and the same optimal boundary control as solving the reducednorder model which was performed by the POD method in 17.07 seconds with 5 nodes in the branch-and-bound tree and 8 solved linear-quadratic

${ }^{1}$ http://www. wetterdienst. de/Deutschlandwette r/Konstanz_Universitaetsstadt/Aktuell; access on May 5, 2017 


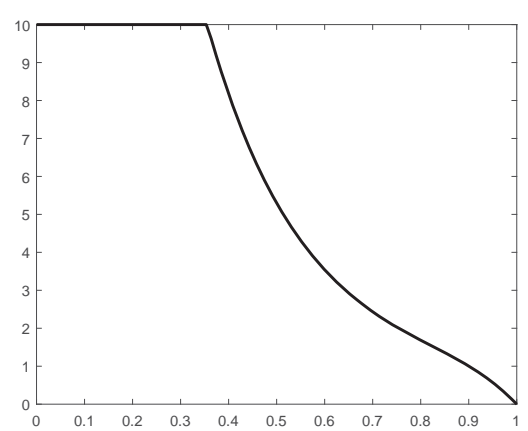

Fig. 3 Run 1: Optimal distributed control $u^{\mathrm{c}}$ over time.

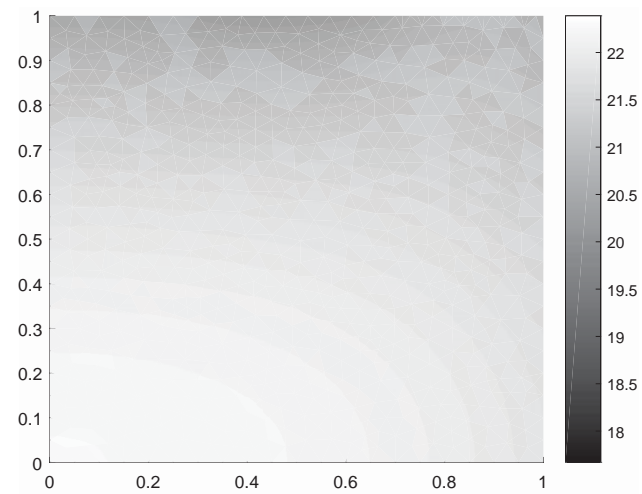

Fig. 4 Run 1: Temperature $y$ at final time $T=1$ in $\Omega$.

optimal control problems. For each node it was sufficient to choose $\ell=4$ in order to satisfy (16), but in nodes 2, 3 and 5 the a-posteriori estimator was larger than 1 and thus we resolved these with an enlarged POD basis of rank 6 . The optimal boundary control is a quite good insulation $\bar{u}^{\mathrm{i}}=(1,2,3)$ and the optimal distributed control (see Fig. 3) displays the heating during the day. In the beginning we have to heat at maximum as the initial temperature is only $18^{\circ}$, during the day it is sufficient to heat less especially as then the outside temperature rises. The heat distribution at the final time $T$ is shown in Fig. 4. It seems sufficiently warm and colder near the window.

In Fig. 5 we can observe that it is the coldest near the window (solid line) where the temperature also falls below the initial $18^{\circ} \mathrm{C}$ in the beginning and reaches its maximum at approximately $21^{\circ} \mathrm{C}$. In the lower left corner where the inner walls meet (dashed line) it is the warmest and from noon on the temperature is already above the desired $22^{\circ} \mathrm{C}$. Also we can notice it is warmer in the middle of the room (dash-dot line) than in the middle of the right outer wall (dotted line) where it is remarkably warmer than at the window due to the better insulation.

Fig. 6 shows the first POD basis element $\psi_{1}^{h}$ which captures the main information about the heat distribution, warmer in the lower-left corner and colder in the up-right area.

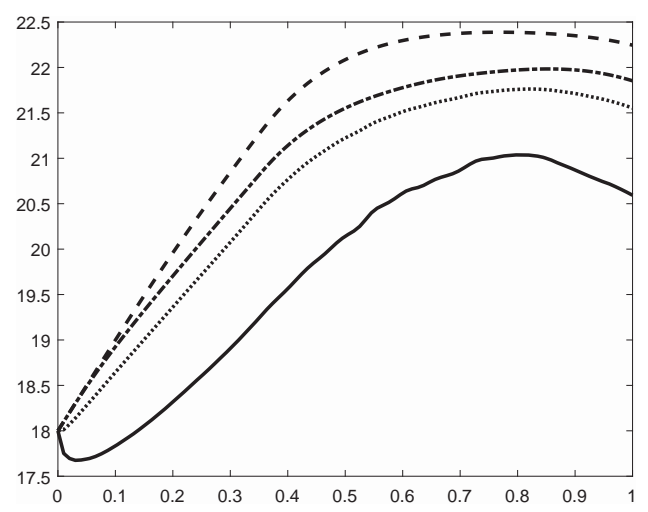

Fig. 5 Run 1: Temperature during the day at certain points in $\Omega$, near the window (solid line), in the middle of the right-outer wall $\left(x_{2} \approx 0.5\right.$, dotted line), in the middle of the room (dash-dot line) and in the lowerleft corner (dashed line).

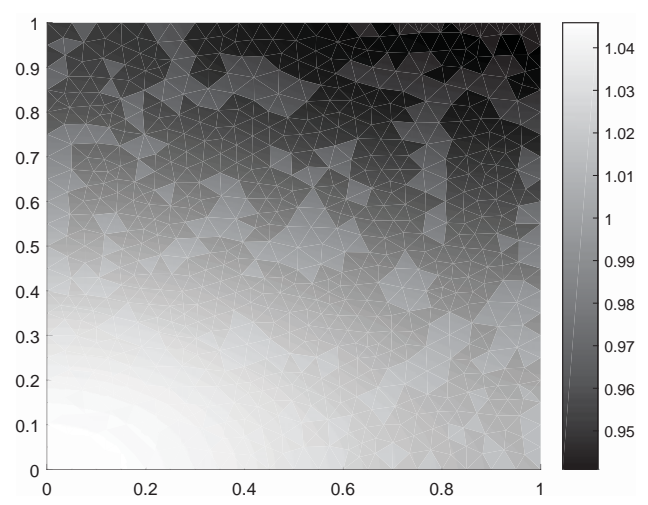

Fig. 6 Run 1: First POD basis element $\psi_{1}^{h}$.

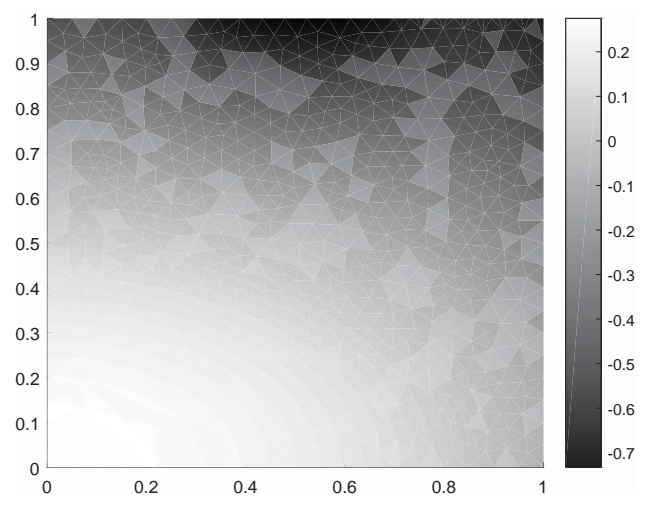

Fig. 7 Run 1: Second POD basis element $\psi_{2}^{h}$.

The second POD basis element Fig. 7 clearly shows the location of the window where it is cold and again the warm lower-left corner. In the third POD basis element Fig. 8 we see a warm up-right corner where the optimal insulation was very $\operatorname{good}\left(u_{1}^{\mathrm{i}}=1\right)$. The further POD basis elements are higher in frequency already; cf. Fig. 9-Fig. 11. We illustrate search tree as it was built during the branch-and-bound algorithm for this example and discuss a few nodes. The numbers represent the order in which they were chosen according to 


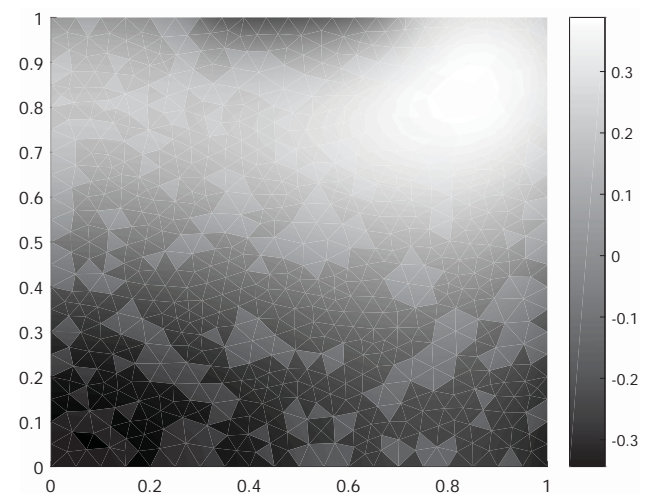

Fig. 8 Run 1: Third POD basis element $\psi_{3}^{h}$.

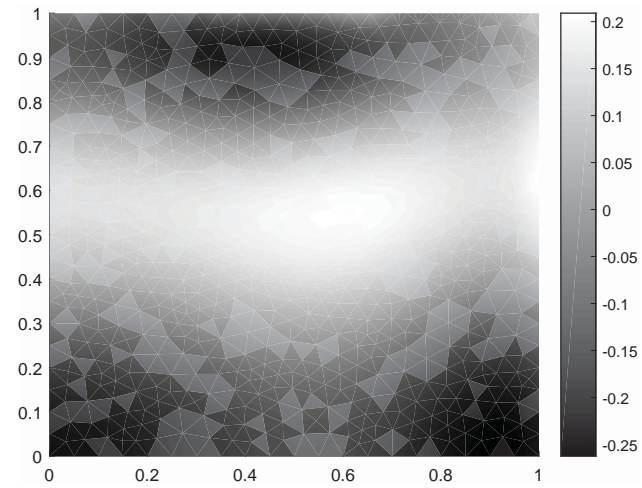

Fig. 9 Run 1: Fourth POD basis element $\psi_{4}^{h}$.

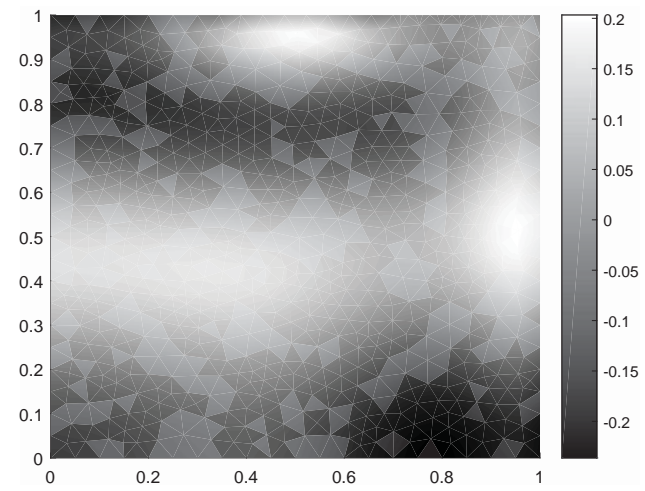

Fig. 10 Run 1: Fifth POD basis element $\psi_{5}^{h}$.

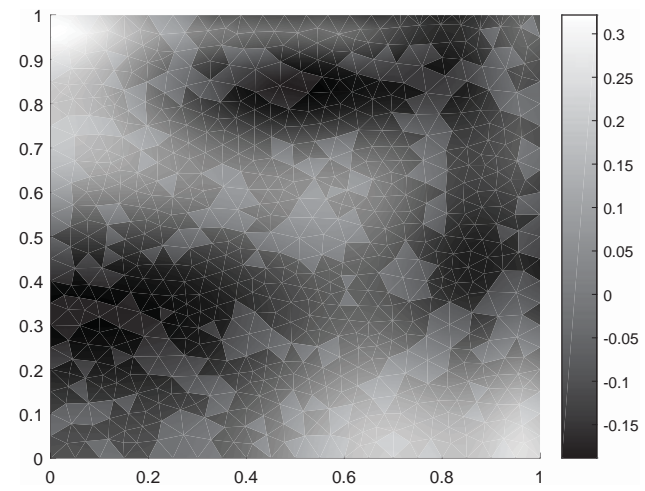

Fig. 11 Run 1: Sixth POD basis element $\psi_{6}^{h}$.

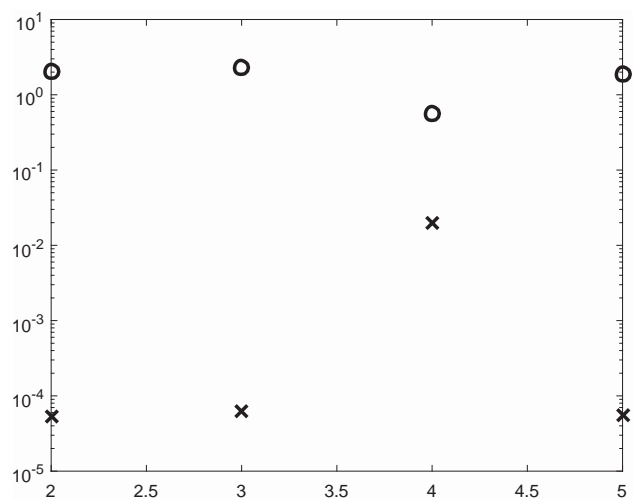

Fig. 12 Run 1: Error between high- and low-dimensional solution (cross) and the a-posteriori estimator (circle) at each of the treated nodes 2 to 5 . The parent node was solved fully as well for the POD method.

\begin{tabular}{c|c} 
node & $\left|\hat{J}^{h}(\bar{u})-\hat{J}^{h}\left(\bar{u}^{\ell}\right)\right|$ \\
\hline 2 & $1.0410 \mathrm{e}-05$ \\
3 & $1.2020 \mathrm{e}-05$ \\
4 & $0.2800 \mathrm{e}-02$ \\
5 & $9.6805 \mathrm{e}-06$
\end{tabular}

Table 1 Run 1: Difference in cost function values for FE-optimal control and POD-FE-optimal control.

the two-phase method and the left node denotes the downchild and the right node the up-child.

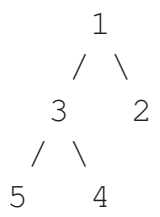

Solving the parent node (1) with $u_{\mathrm{a}}^{\mathrm{i}}=(1,2,1)$ and $u_{\mathrm{b}}^{\mathrm{i}}=$ $(10,10,10)$ gives the optimal solution $(1,2,3.8668)$ with cost functional value $\hat{J}^{h}=35.4414$. As the third component violates the integrality constraint it is the only branching candidate for which both child nodes are solved according to the strong branching method. The next node to be explored is due to the two-phase method the up-child (2) with lower bound $a=(1,2,4)$ whose solution $(1,2,4)$ is integer with cost functional value 35.4724 giving the current global upper bound. After a few more steps - solving the down-child (3), branching on variable $u_{2}^{\mathrm{i}}$ and solving the two arising children (4) and (5) - we found the optimal solution in the node (5) with lower cost functional value 35.3356 and optimal integer solution $(1,2,3)$.

For comparison we solved all nodes as well without the model reduction and computed the error $\left\|\bar{u}-\bar{u}^{\ell}\right\|_{u}$ which is plotted alongside the a posteriori estimator in Fig. 12. In Tab. 1 we dispayed the difference in the objective for the optimal control and the suboptimal POD control at each node.

Splitting the domain into four heating tiles, i.e. $N^{\mathrm{c}}=4$, yields the optimal integer solution $u^{\mathrm{i}}=(1,2,1)$ when solving the 


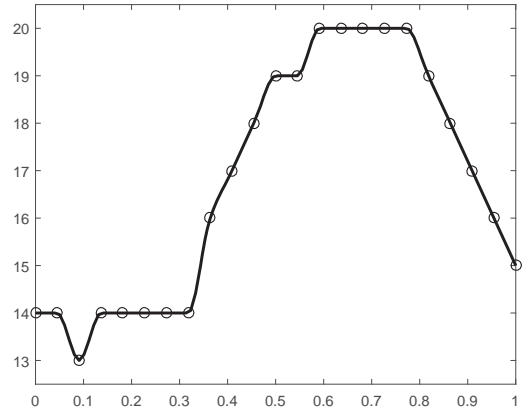

Fig. 13 Run 2: Weather data: hourly Lisbon forecast (circles) on May 6th and cubic interpolation (solid line).

parent node within 12.55 seconds already. That means the outside temperature is so cold that instantly the best insulation is chosen and the optimization terminates without the need of the branch-and-bound algorithm. Hence we continue with another example.

7.2 Weather data from Lisbon, Portugal

Longing for some warmer weather we choose an outside temperature $y_{a}$ from Lisbon, Portugal, ranging from $13^{\circ}$ to $21^{\circ} \mathrm{C}$, see Fig. $13^{2}$ based on the hourly weather forecast on May 6th.

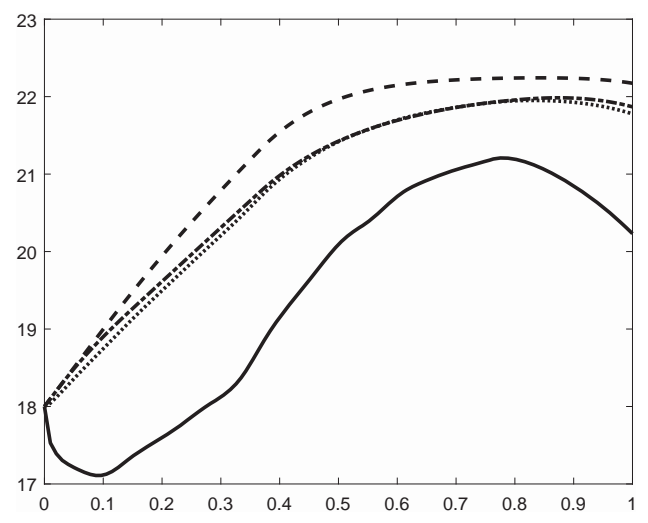

Fig. 14 Run 2: Temperature at certain points in the room over time, near the window (solid), in the middle of the right-outer wall (dotted), in the middle of the room (dash-dotted) and in the lower left corner (dashed line).

Run 2. With the POD method the problem was solved within 21.69 seconds (compared to 48.34 seconds for $\left(\hat{\mathbf{P}}^{h}\right)$ ) with 6 nodes in the branch-and-bound tree and 16 relaxations solved with usually four basis elements. In nodes 2, 4, 5 and 6 the a-posteriori estimate was larger than the tolerance of 1 , thus we resolved these nodes with 6 basis elements. The resulting optimal control is $u^{\mathrm{i}}=(1,5,8)$, i.e. the insulation material is a lot cheaper than it had to be for the colder outside temperature in Konstanz, which conveniently meets our expectations. Looking at Fig. 14 we see that it is again the coldest near the window and the warmest in the lower left corner. As the first boundary segment has a very good insulation, we can observe similar temperatures in the middle of the room (dash-dotted line) and at the middle of the right outer wall (dotted). Compared to Fig. 5 the cheaper insulation leads to an increased loss of warmth near the window, especially in the beginning. The branch-and-bound tree looks like this:

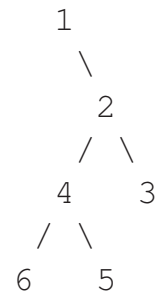

The down-child of the parent node was solved during the strong branching algorithm, but then pruned as its cost functional value was greater than the global upper bound for the integer valued solution found in the up-branch. Nodes 3, 5 and 6 all gave integer-valued solutions, the optimal one was found in node 6 .

Run 3. We consider the Lisbon outside temperature as in the previous run, but with four heating tiles now. The com-

2 https: / / www . timeanddate. com/weather/portuga I/Iisbon; access on May 6, 3027 


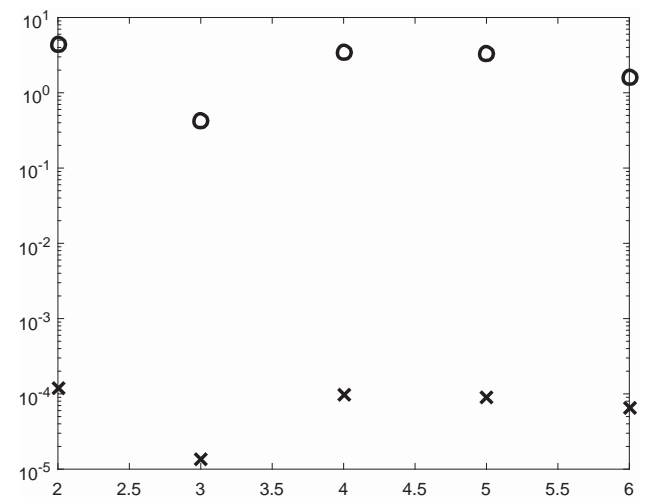

Fig. 15 Run 2: Error (x) and estimator (o). At node 2, 4, 5 and 6 the basis got updated, i.e. two additional basis vectors were used to resolve the node.

\begin{tabular}{c|c} 
node & $\left|\hat{J}^{h}\left(u^{F E}\right)-\hat{J}^{h}\left(u^{P O D}\right)\right|$ \\
\hline 2 & $8.5704 \mathrm{e}-05$ \\
3 & $4.9662 \mathrm{e}-07$ \\
4 & $1.9766 \mathrm{e}-05$ \\
5 & $2.9137 \mathrm{e}-05$ \\
6 & $2.1637 \mathrm{e}-05$
\end{tabular}

Table 2 Run 2: Difference in cost function values for FE-optimal control and POD-FE-optimal control.

\begin{tabular}{c|c} 
node & $\left|\hat{J}^{h}\left(u^{F E}\right)-\hat{J}^{h}\left(u^{P O D}\right)\right|$ \\
\hline 2 & $3.0322 \mathrm{e}-05$ \\
3 & $6.1947 \mathrm{e}-05$ \\
4 & $2.4099 \mathrm{e}-08$
\end{tabular}

Table 3 Run 3: Difference in cost function values for FE-optimal control and POD-FE-optimal control.

putational time was 14.48 seconds for the POD method and 31.23 seconds for the FE method. The branch-and-bound algorithm needs 4 nodes and 10 linear-quadratic problems were solved. The basis got updated from 4 to 7 basis elements as the a-posteriori estimate was larger than required tolerance in nodes 2 and 3 . The resulting optimal boundary control is $u^{\mathrm{i}}=(1,4,6)$ and the optimal time-dependent distributed control $u^{\mathrm{c}}$ is displayed in Fig. 16 and in a closer view inFig. 17.

All heating tiles have to heat at maximum in the beginning to get near the desired temperature and less during the day when the room is already warm and also the outside temperature is higher. The lower-left heating tile $u_{1}^{\mathrm{c}}$ (solid) and $u_{2}^{\mathrm{c}}$ (dashed) have to heat less than the upper-right $u_{3}^{\mathrm{c}}$ (dashdot) where we have half of the window but a good insulation $\left(u_{1}^{\mathrm{i}}=1\right)$ and upper-left $u_{4}^{\mathrm{c}}$ (dotted), where we have a half of the window and the cheapest insulation $\left(u_{3}^{i}=6\right)$ and consequently, have to heat the most.

In Fig. 18 we can observe that even at the warmest point in the room, the lower-left corner, we barely reach $21.5^{\circ} \mathrm{C}$ which is significantly lower than it was in the second run in which we had one heating tile. This is because we did not consider the size of the heating tiles in our cost functional

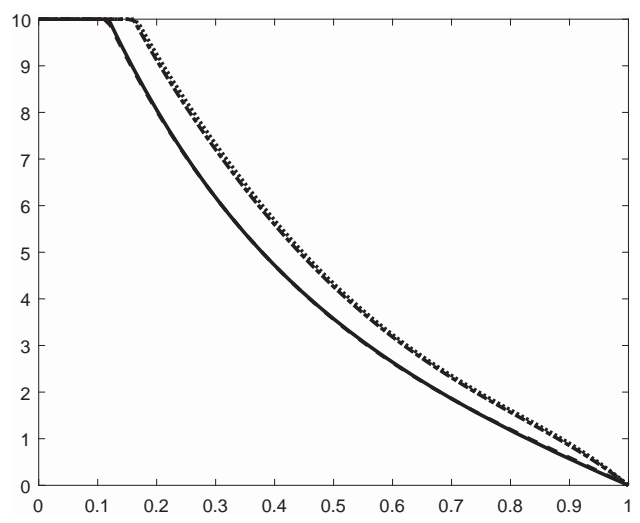

Fig. 16 Run 3: Distributed controls over time, the two heating tiles in the lower half have to heat less than the two in the upper half of the room.

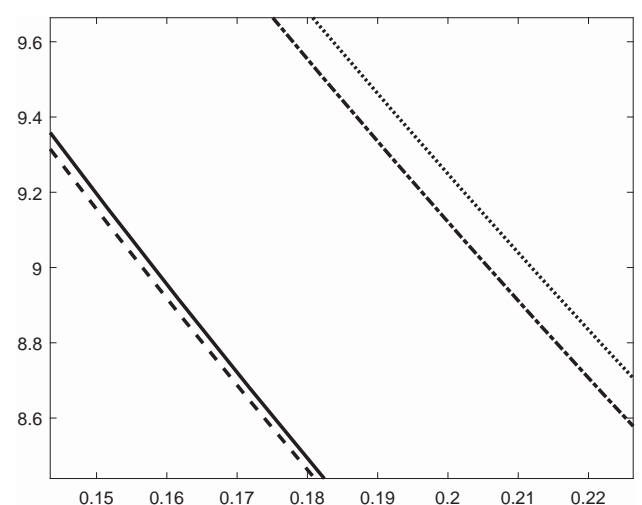

Fig. 17 Run 3: A closer look at the distributed controls over time: lower-left $u_{1}^{\mathrm{c}}$ (solid) and lower-right $u_{2}^{\mathrm{c}}$ (dashed), upper-right $u_{3}^{\mathrm{c}}$ (dashdot) and upper-left $u_{4}^{\mathrm{c}}$ (dotted).

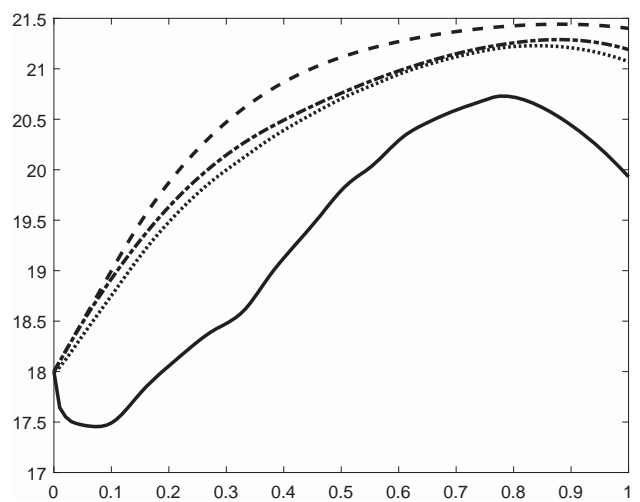

Fig. 18 Run 3: Temperature at certain points in the room, the window (solid), in the middle of the right outer wall (dotted), in the middle (dash-dot) and in the lower left corner (dashed).

which means as we kept $\alpha^{\mathrm{c}}=0.3$ for all four heating tiles, the heating costs were four times higher.

Run 4. Hence, choosing all four $\alpha^{\mathrm{c}}=0.075$ gives similar temperatures as in Fig. 14 and and a similar optimal $u^{\mathrm{i}}=$ $(1,6,7)$ (compared to $u^{\mathrm{i}}=(1,5,8)$ in the one-tile case). The computation required 21.44 seconds with 7 nodes and 15 


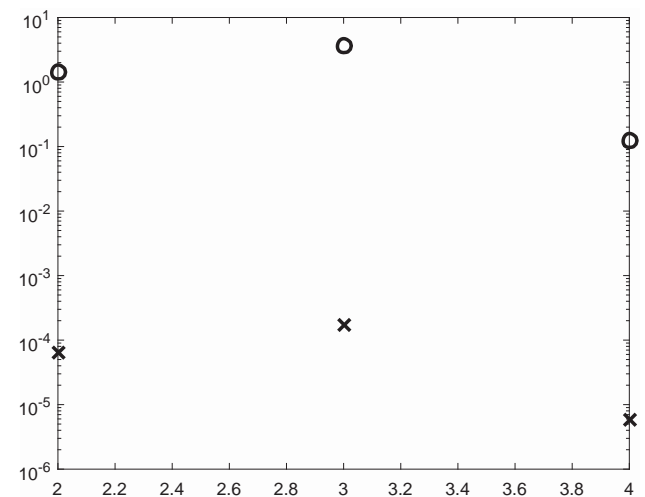

Fig. 19 Run 3: Error (x) and estimator (o) at each of the solved nodes, $\ell=7$ for nodes 2 and 3 and $\ell=4$ in the last node.

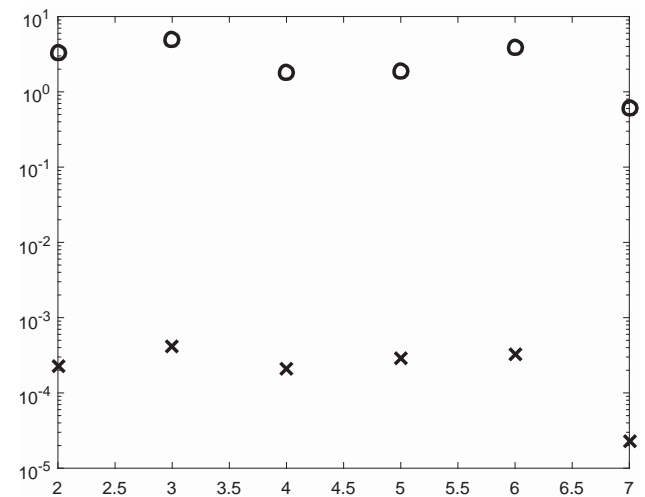

Fig. 20 Run 4: Error (x) and estimator (o) at each of the solved nodes for $\alpha^{c}=0.075$. The basis was updated at nodes 2 to 6 from 4 to 9 basis elements in order to satisfy the increased energy bound.

\begin{tabular}{c|c} 
node & $\left|\hat{J}^{h}\left(u^{F E}\right)-\hat{J}^{h}\left(u^{P O D}\right)\right|$ \\
\hline 2 & $8.9129 \mathrm{e}-05$ \\
3 & $1.4692 \mathrm{e}-04$ \\
4 & $2.5850 \mathrm{e}-05$ \\
5 & $9.2066 \mathrm{e}-05$ \\
6 & $8.2975 \mathrm{e}-05$ \\
7 & $2.3617 \mathrm{e}-07$
\end{tabular}

Table 4 Run 4: Difference in cost function values for FE-optimal control and POD-FE-optimal control.

optimization problems solved.

Run 5. Another option would be to punish the difference to the desired temperature more, for example we took $\alpha^{Q}=20$ instead of 10. As displayed in Fig. 21 the temperature difference within the room is significantly smaller and the chosen optimal insulation is $u^{i}=(1,2,5)$ is a better one since heating is more expensive again $\left(\alpha^{c}=0.3\right.$ for all heating tiles). The elapsed time was 17.45 seconds with 4 nodes in the branch-and-bound tree and 12 relaxations solved.. In all nodes the a-posteriori estimate was too large for $\ell=3$, so we took 6 basis elements.

Run 6. As well we try the one-tile Lisbon situation with an increased $\alpha^{Q}=20$ and the error alongside with the a pos-

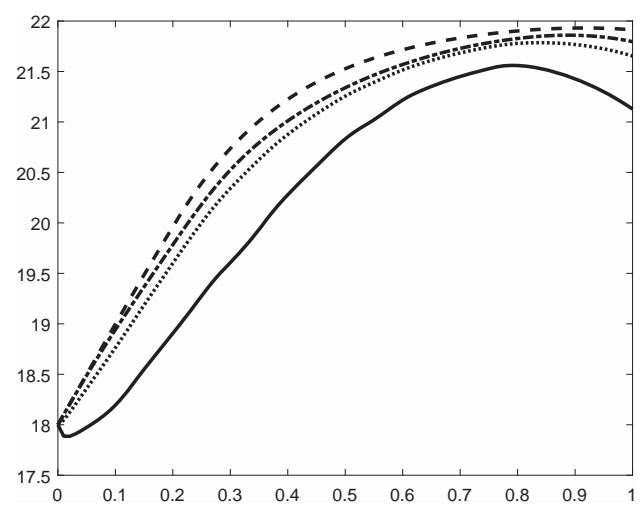

Fig. 21 Run 5: Temperature at certain points in the room, the window (solid), in the middle of the right outer wall (dotted), in the middle (dash-dot) and in the lower left corner (dashed).

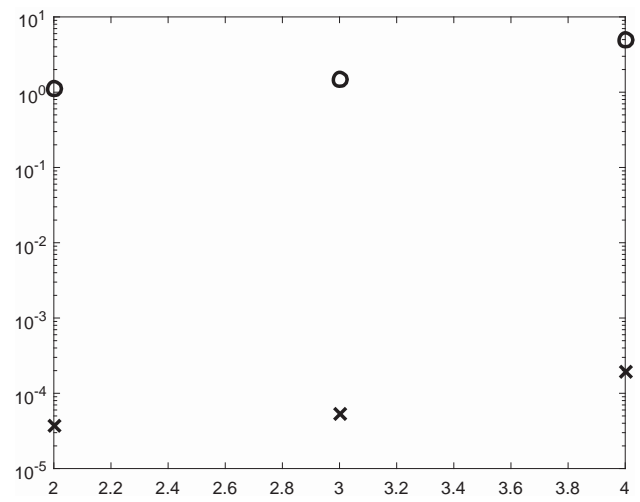

Fig. 22 Run 5: Error (x) and estimator (o) at each of the solved nodes for $\alpha^{Q}=20$.

\begin{tabular}{c|c} 
node & $\left|\hat{J}^{h}\left(u^{F E}\right)-\hat{J}^{h}\left(u^{P O D}\right)\right|$ \\
\hline 2 & $2.7761 \mathrm{e}-05$ \\
3 & $7.1499 \mathrm{e}-06$ \\
4 & $9.2719 \mathrm{e}-05$
\end{tabular}

Table 5 Run 5: Difference in cost function values for FE-optimal control and POD-FE-optimal control.

\begin{tabular}{c|c} 
node & $\left|\hat{J}^{h}\left(u^{F E}\right)-\hat{J}^{h}\left(u^{P O D}\right)\right|$ \\
\hline 2 & $2.7603 \mathrm{e}-05$ \\
3 & $1.0342 \mathrm{e}-04$ \\
4 & $1.8425 \mathrm{e}-06$ \\
5 & $5.3310 \mathrm{e}-05$ \\
6 & $3.4490 \mathrm{e}-05$ \\
7 & $4.2517 \mathrm{e}-05$
\end{tabular}

Table 6 Run 6: Difference in cost function values for FE-optimal control and POD-FE-optimal control.

teriori estimator can be seen in Fig. 23. The computation required 25.81 seconds where the branch-and-bound algorithm had to explore 7 nodes in which 17 relaxations were solved in total with $\ell=5$ in all nodes. The optimal boundary control is $u^{i}=(1,4,5)$ which means a better insulation as in Run 2 (where we chose $u^{i}=(1,5,8)$ for a lower $\alpha^{Q}=10$ ). 


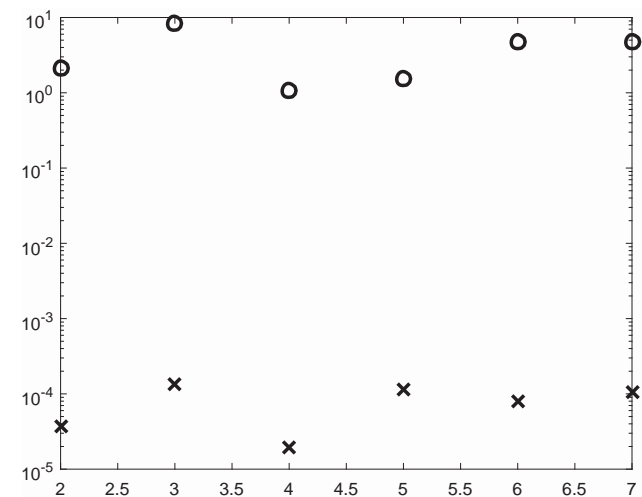

Fig. 23 Run 6: Error (x) and estimator (o) at each of the solved nodes for $\alpha^{Q}=20$.

\begin{tabular}{c|c|c|c|c} 
Run & FE time & POD time & \# nodes & \# relaxations \\
\hline 1 & 28.37 & 17.07 & 5 & 8 \\
2 & 48.34 & 21.69 & 6 & 16 \\
3 & 31.23 & 14.48 & 4 & 10 \\
4 & 45.80 & 21.44 & 7 & 15 \\
5 & 37.78 & 17.45 & 4 & 12 \\
6 & 47.02 & 25.81 & 7 & 17
\end{tabular}

Table 7 Comparison of computational costs for solving the highdimensional model $\left(\hat{\mathbf{P}}^{h}\right)$ and the low-dimensonal POD-based model. For the latter the number of explored nodes and solved relaxations is denoted.

\subsection{Comparison of the computational cost}

In Tab. 7 we can see that the POD method is usually twice as fast, and both computational times depend on the number of explored nodes. The FE method might sometimes need to solve less relaxations, as some of the nodes were resolved in the POD method with a larger POD basis.

\section{Conclusion and Outlook}

In this work, we have applied a standard Branch-and-Bound algorithm to a linear-quadratic Mixed-Integer Optimal Control Problem (MIOCP) which was motivated by the physical challenge of heating a room which is subject to heat loss through the exterior walls. The distributed controls representing an underfloor heating were chosen as continuous and time-dependent, whereas the boundary controls were only allowed to take discrete values, thereby indicating a certain choice of insulation material. By branching repeatedly on these latter control variables, it became necessary to repeatedly solve a relaxed version of the MIOCP which is just a standard linear-quadratic optimal control problem. We have employed a POD-Galerkin ansatz for this relaxed problem where the initial data is collected by optimizing once on the mother node of the Branch-and-Bound tree. Replacing the full problem with this reduced-order surrogate for all subsequent nodes has shown to lead to a speedup in all numerical experiments. Even though this speedup only turned out to be of a factor 2, what was more significant was the observation that this is a strategy which appears to work qualitatively. We are also confident that a much higher speedup can be achieved by turning towards more complex models which require more solves of the state equation. Such increased complexity could arise for finer spatial and time grids, a higher number of discrete control variables leading to bigger Branch-and-Bound trees, or even more detailed models to describe the temperature distribution like an advectiondiffusion-reaction equation or the Boussinesq equation.

Furthermore, we have presented a straightforward estimator indicating the error between the optimal and PODsuboptimal control. Numerical tests have validated the fact that this estimator bounds the real error from above, even though it overshoots it by several orders of magnitude. In any case, the estimator was used as an indicator for the quality of the reduced-order model to possibly update the reducedorder model if it exceeded a certain tolerance. For future work, it may be beneficial to investigate where this drastic overestimation comes from and how it can be placed in check. In [3], a heuristic was deployed in which real error and estimator for some random points were calculated offline before the optimization in order to and gauge the overestimation factor.

A similar strategy could be employed here: Since the optimal solution $\bar{u} \in \mathcal{U}_{\mathrm{ad}}$ is computed in high fidelity anyway, we could define some sample controls $u \in \mathcal{U}_{\text {ad }}$ and compute the error estimator estimator as well as the true error for these controls. Looking at the resulting optimizers to the problem, we have observed in several test runs that a physically and economically reasonable behavior is reflected in the solutions. The trade-off between the cost of high insulation and the resulting benefit of low temperature transmission to the outside can be chosen by varying the parameters of the model. All in all, it could be observed that the algorithm reliably allowed us to solve the problem and that the problem qualitatively depicts the physical behavior of the temperature in the room.

A first step in future work would definitely be to consider an increase in the number of discrete variables to the system. Right now, the low number of three of these leads to quite small Branch-and-Bound trees which are solved rather rapidly. The true time-saving possibilities of this strategy will only show when considering larger problems with more state and adjoint equations to be solved. Furthermore, a natural next step that will also challenge the way POD can perform is to consider an advection term in the state equation, leading to more dynamic solutions and thus requiring more basis functions in the surrogate model. Lastly, the current insulation model could be replaced by $y(t, s)=u^{\mathrm{i}}\left(y_{a}(t)-\right.$ $y(t, s))$ for $(s, t) \in \Sigma$ with an outside temperature $y_{a}:[0, T] \rightarrow$ $\mathbb{R}$. This would lead to a model that are no longer linear in the binary control variable and thus harder to solve. Some work 
has already been done for optimal control of these kinds of models in [2] and could be readily applied to this setting.

Acknowledgements This work is supported by the BMWi-project $H y$ brides Planungsverfahren zur energieeffizienten Wärme- und Stromversorgung von städtischen Verteilnetzen funded by the German Ministry for Economic Affairs and Energy.

\section{References}

1. Arada, N., Casas, E., Tröltzsch, F.: Error estimates for the numerical approximation of a semilinear elliptic control problem. Computational Optimization and Applications 23, 201-229 (2002)

2. Banholzer, S., Beermann, D.: Optimal control and model-order reduction of an abstract parabolic system containing a controlled bilinear form. Tech. rep., University of Konstanz (2016)

3. Beermann, D., Dellnitz, M., Peitz, S., Volkwein, S.: Set-oriented multiobjective optimal control of pdes using proper orthogonal decomposition. https://kops.unikonstanz.de/handle/123456789/38752 (2017). Submitted

4. Benner, P., Sachs, E., Volkwein, S.: Model order reduction for pde constrained optimization. In: Trends in PDE Constrained Optimization, International Series of Numerical Mathematics, pp. 303-326. Birkhäuser, Basel (2014)

5. Casas, E., Tröltzsch, F.: Error estimates for the finite-element approximation of a semilinear elliptic control problem. Control and Cybernetics 31, 695-712 (2002)

6. Dautray, R., Lions, J.L.: Mathematical Analysis and Numerical Methods for Science and Technology. Volume 5: Evolution Problems I. Springer-Verlag, Berlin (2000)

7. EPBD: On the energy performance of buildings. Official Journal of the European Union (2010). Directive 2010/31/EU of the European Parliament and of the Council

8. Evans, L.: Partial Differential Equations. American Math. Society, Providence, Rhode Island (2008)

9. Foucquier, A., Robert, S., Suard, F., Stephan, L., Jay, A.: State of the art in building modelling and energy performance prediction: A review. Renewable and Sustainable Energy Reviews 23, 272288 (2013)

10. Fügenschuh, A., Geißler, B., Martin, A., Morsi, A.: The transport PDE and $\mathrm{m}+$ mixed-integer linear programming. In: Models and Algorithms for Optimization in Logistics, 21.06. - 26.06.2009 (2009)

11. Gerdts, M.: Optimal Control of ODEs and DAEs. De Gruyter (2011)

12. Grimm, E., Gubisch, M., Volkwein, S.: Numerical analysis of optimality-system pod for constrained optimal control. Lecture Notes in Computational Science and Engineering 105, 297-317 (2015)

13. Gubisch, M., Volkwein, S.: Proper orthogonal decomposition for linear-quadratic optimal control. To appear in P. Benner, A. Cohen, M. Ohlberger, and K. Willcox (eds.), Model Reduction and Approximation: Theory and Algorithms. SIAM, Philadelphia, PA (2017)

14. Gugat, M., Leugering, G., Martin, A., Schmidt, M., Sirvent, M., Wintergerst, D.: MIP-based instantaneous control of mixedinteger PDE-constrained gas transport problems (2017)

15. Hante, F.: Relaxation methods for hyperbolic PDE mixed-integer optimal control problems. Optimal Control Applications and Methods (2017). To appear

16. Hante, F., Sager, S.: Relaxation Methods for Mixed-Integer Optimal Control of Partial Differential Equations. Computational Optimization and Applications 55(1), 197-225 (2013)
17. Hinze, M., Volkwein, S.: Error estimates for abstract linearquadratic optimal control problems using proper orthogonal decomposition. Computational Optimization and Application 39, 319-345 (2008)

18. Holmes, P., Lumley, J., Berkooz, G., Rowley, C.: Turbulence, Coherent Structures, Dynamical Systems and Symmetry. Cambridge Monographs on Mechanics. Cambridge University Press, Cambridge, 2nd ed. (2012)

19. Land, A.H., Doig, A.G.: An automatic method of solving discrete programming problems. Econometrica 26, 497-520 (1960)

20. Leyffer, S., Cay, P., Kouri, D., van Bloemen Waanders, B.: Mixedinteger PDE-constrained optimization. Tech. rep., Oberwolfach Report (2015)

21. Malanowski, K., Büskens, C., Maurer, H.: Convergence of approximations to nonlinear control problems. In: Fiacco, A.V. (ed.) Mathematical Programming with Data Perturbation, Marcel Dekker, New York pp. 253-284 (1997)

22. Sachs, E., Volkwein, S.: POD Galerkin approximations in PDEconstrained optimization. GAMM-Mitteilungen 33, 194-208 (2010)

23. Sager, S., Bock, H., Diehl, M.: The integer approximation error in mixed-integer optimal control. Mathematical programming 133, $1-23$ (2012)

24. Schilders, W., van der Vorst, H., Rommes, J.: Model Order Reduction: Theory, Research Aspects and Applications. The European Consortium for Mathematics in Industry. Springer (2008)

25. Singler, J.: New POD expressions, error bounds, and asymptotic results for reduced order models of parabolic PDEs. SIAM Journal on Numerical Analysis 52, 852-876 (2014)

26. Studinger, A., Volkwein, S.: Numerical analysis of POD aposteriori error estimation for optimal control. International Series of Numerical Mathematics 164, 137-158 (2013)

27. Sunder, S., Dion, J.: Federal research and development agenda for net-zero energy, high-performance green buildings. Tech. rep., National Science and Technology Council, Committee on Technology, Subcommittee on Buildings Technology Research and Development (2008)

28. Tröltzsch, F.: Optimal Control of Partial Differential Equations, vol. 112. American Mathematical Society (2010)

29. Tröltzsch, F., Volkwein, S.: POD a-posteriori error estimates for linear-quadratic optimal control problems. Computational Optimization and Applications 44, 83-115 (2009)

30. Vanderlei, R.: Linear Programming. Foundations and Extensions. International Series in Operations Research \& Management Science. Springer (2014)

31. WBCSD: Energy E+efficiency in buildings: business realities and opportunities. Tech. rep., World Business Council for Sustainable Development (2007)

32. WBCSD: Energy efficiency in buildings: transforming the market. Tech. rep., World Business Council for Sustainable Development (2009) 